\title{
Equalities between the BLUEs and BLUPs under the partitioned linear fixed model and the corresponding mixed model
}

\author{
Stephen J. Haslett, Jarkko Isotalo, and Simo Puntanen
}

\begin{abstract}
In this article we consider the partitioned fixed linear model $\mathscr{F}: \mathbf{y}=\mathbf{X}_{1} \boldsymbol{\beta}_{1}+\mathbf{X}_{2} \boldsymbol{\beta}_{2}+\boldsymbol{\varepsilon}$ and the corresponding mixed model $\mathscr{M}: \mathbf{y}=$ $\mathbf{X}_{1} \boldsymbol{\beta}_{1}+\mathbf{X}_{2} \mathbf{u}+\boldsymbol{\varepsilon}$, where $\boldsymbol{\varepsilon}$ is a random error vector and $\mathbf{u}$ is a random effect vector. In 2006, Isotalo, Möls, and Puntanen found conditions under which an arbitrary representation of the best linear unbiased estimator (BLUE) of an estimable parametric function of $\boldsymbol{\beta}_{1}$ in the fixed model $\mathscr{F}$ remains BLUE in the mixed model $\mathscr{M}$. In this paper we extend the results concerning further equalities arising from models $\mathscr{F}$ and $\mathscr{M}$.
\end{abstract}

\section{Introduction}

Let the partitioned linear fixed effects model be

$$
\mathscr{F}=\left\{\mathbf{y}, \mathbf{X}_{1} \boldsymbol{\beta}_{1}+\mathbf{X}_{2} \boldsymbol{\beta}_{2}, \mathbf{V}\right\}=\{\mathbf{y}, \mathbf{X} \boldsymbol{\beta}, \mathbf{V}\},
$$

i.e., the $n$-dimensional observable random vector $\mathbf{y}$ is of the form

$$
\mathbf{y}=\mathbf{X}_{1} \boldsymbol{\beta}_{1}+\mathbf{X}_{2} \boldsymbol{\beta}_{2}+\varepsilon, \quad \operatorname{cov}(\varepsilon)=\mathbf{V}, \mathrm{E}(\varepsilon)=\mathbf{0},
$$

where $\mathbf{X}_{1} \in \mathbb{R}^{n \times p_{1}}$ and $\mathbf{X}_{2} \in \mathbb{R}^{n \times p_{2}}$ are known matrices, $p_{1}+p_{2}=p$, $\boldsymbol{\beta}_{i} \in \mathbb{R}^{p_{i}}, i=1,2$, are vectors of unknown fixed effects. The covariance matrix $\mathbf{V}$ of the random error vector $\varepsilon$ is assumed to be known.

Consider the linear mixed model $\mathscr{M}$ which is obtained from $\mathscr{F}$ by replacing the fixed vector $\boldsymbol{\beta}_{2}$ with the random effect vector $\mathbf{u}$ :

$$
\mathscr{M}: \mathbf{y}=\mathbf{X}_{1} \boldsymbol{\beta}_{1}+\mathbf{X}_{2} \mathbf{u}+\varepsilon, \quad \operatorname{cov}(\varepsilon)=\mathbf{V}, \mathrm{E}(\varepsilon)=\mathbf{0},
$$

Received March 29, 2021.

2020 Mathematics Subject Classification. 62J05, 62J10.

Key words and phrases. Best linear unbiased estimator, best linear unbiased predictor, BLUE, BLUP, equality of the BLUEs, partitioned linear model.

https://doi.org/10.12697/ACUTM.2021.25.16

Corresponding author: Simo Puntanen 
where $\mathbf{X}_{1}$ and $\mathbf{X}_{2}$ are as in $\mathscr{F}, \boldsymbol{\beta}_{1}$ is a vector of unknown fixed effects, $\mathbf{u}$ is an unobservable vector of random effects with $\mathrm{E}(\mathbf{u})=\mathbf{0}, \operatorname{cov}(\mathbf{u})=\mathbf{D}$, $\operatorname{cov}(\varepsilon, \mathbf{u})=\mathbf{0} ; \mathbf{V}$ and $\mathbf{D}$ are assumed to be known. In this situation we have

$$
\begin{gathered}
\operatorname{cov}\left(\begin{array}{l}
\varepsilon \\
\mathbf{u}
\end{array}\right)=\left(\begin{array}{cc}
\mathbf{V} & \mathbf{0} \\
\mathbf{0} & \mathbf{D}
\end{array}\right), \quad \operatorname{cov}\left(\begin{array}{l}
\mathbf{y} \\
\mathbf{u}
\end{array}\right)=\left(\begin{array}{cc}
\mathbf{\Sigma} & \mathbf{X}_{2} \mathbf{D} \\
\mathbf{D} \mathbf{X}_{2}^{\prime} & \mathbf{D}
\end{array}\right), \\
\operatorname{cov}(\mathbf{y})=\operatorname{cov}\left(\mathbf{X}_{2} \mathbf{u}+\varepsilon\right)=\mathbf{\Sigma}=\mathbf{X}_{2} \mathbf{D} \mathbf{X}_{2}^{\prime}+\mathbf{V} .
\end{gathered}
$$

Notice that under $\mathscr{F}$ we have $\operatorname{cov}(\mathbf{y})=\mathbf{V}$ but under $\mathscr{M}, \operatorname{cov}(\mathbf{y})=\mathbf{\Sigma}$.

As for notation, $\mathrm{r}(\mathbf{A}), \mathbf{A}^{-}, \mathbf{A}^{+}, \mathscr{C}(\mathbf{A})$, and $\mathscr{C}(\mathbf{A})^{\perp}$, denote, respectively, the rank, a generalized inverse, the (unique) Moore-Penrose inverse, the column space, and the orthogonal complement of $\mathscr{C}(\mathbf{A})$. By $\mathbf{A}^{\perp}$ we denote any matrix satisfying $\mathscr{C}\left(\mathbf{A}^{\perp}\right)=\mathscr{C}(\mathbf{A})^{\perp}$. Furthermore, we will write $\mathbf{P}_{\mathbf{A}}=$ $\mathbf{A} \mathbf{A}^{+}=\mathbf{A}\left(\mathbf{A}^{\prime} \mathbf{A}\right)^{-} \mathbf{A}^{\prime}$ to denote the orthogonal projector onto $\mathscr{C}(\mathbf{A})$. The orthogonal projector onto $\mathscr{C}(\mathbf{A})^{\perp}$ is denoted as $\mathbf{Q}_{\mathbf{A}}=\mathbf{I}_{a}-\mathbf{P}_{\mathbf{A}}$, where $\mathbf{I}_{a}$ refers to the $a \times a$ identity matrix and $a$ is the number of rows of $\mathbf{A}$. We use the short notations

$$
\mathbf{M}=\mathbf{I}_{n}-\mathbf{P}_{\mathbf{X}} \in\left\{\mathbf{X}^{\perp}\right\}, \quad \mathbf{M}_{i}=\mathbf{I}_{n}-\mathbf{P}_{\mathbf{X}_{i}} \in\left\{\mathbf{X}_{i}^{\perp}\right\}, i=1,2 .
$$

Let $\mathbf{K} \in \mathbb{R}^{k \times p}$. Then a linear statistic $\mathbf{A y}$ is said to be a linear unbiased estimator (LUE) for $\mathbf{K} \boldsymbol{\beta}$ in $\mathscr{F}$ if its expectation is equal to $\mathbf{K} \boldsymbol{\beta}$, which happens if and only if $\mathbf{K}^{\prime}=\mathbf{X}^{\prime} \mathbf{A}^{\prime}$; then $\mathbf{K} \boldsymbol{\beta}$ is said to be estimable. The LUE $\mathbf{A y}$ is the best linear unbiased estimator, BLUE, of estimable $\mathbf{K} \boldsymbol{\beta}$ if Ay has the smallest covariance matrix in the Löwner sense among all LUEs of $\mathbf{K} \boldsymbol{\beta}$ :

$$
\operatorname{cov}(\mathbf{A y}) \leq_{\mathrm{L}} \operatorname{cov}\left(\mathbf{A}_{\#} \mathbf{y}\right) \text { for all } \mathbf{A}_{\#} \in \mathbb{R}^{k \times n}: \mathbf{A}_{\#} \mathbf{X}=\mathbf{K} .
$$

Correspondingly, the linear predictor By is said to be unbiased (LUP) for a $q$-dimensional random vector $\mathbf{g}=\mathbf{K}_{1} \boldsymbol{\beta}_{1}+\mathbf{J u}$ under $\mathscr{M}$ if the expected prediction error is zero, i.e., $\mathrm{E}(\mathbf{g}-\mathbf{B y})=\mathbf{0}$ for all $\boldsymbol{\beta}_{1}$; here $\mathbf{K}_{1} \in \mathbb{R}^{q \times p_{1}}$ and $\mathbf{J} \in \mathbb{R}^{q \times p_{2}}$. Now a LUP By is the best linear unbiased predictor, BLUP for $\mathbf{g}$ if it minimizes the covariance matrix of the prediction error among all LUPs, i.e., we have the Löwner ordering

$$
\operatorname{cov}(\mathbf{g}-\mathbf{B y}) \leq_{\mathrm{L}} \operatorname{cov}\left(\mathbf{g}-\mathbf{B}_{\#} \mathbf{y}\right) \quad \text { for all } \mathbf{B}_{\#} \in \mathbb{R}^{q \times n}: \mathbf{B}_{\#} \mathbf{X}_{1}=\mathbf{K}_{1} .
$$

Suppose we are interested in comparing the BLUE of $\mathbf{K}_{1} \boldsymbol{\beta}_{1}$ under $\mathscr{F}$ and $\mathscr{M}$. To do this we have to assume that $\mathbf{K}_{1} \boldsymbol{\beta}_{1}$ is estimable in both models. By Groß and Puntanen [2, Lemma 1], $\mathbf{K}_{1} \boldsymbol{\beta}_{1}$ is estimable under $\mathscr{F}$ if and only if $\mathscr{C}\left(\mathbf{K}_{1}^{\prime}\right) \subseteq \mathscr{C}\left(\mathbf{X}_{1}^{\prime} \mathbf{M}_{2}\right)$, i.e., $\mathbf{K}_{1}=\mathbf{L M}_{2} \mathbf{X}_{1}$ for some matrix $\mathbf{L}$. Thus if we wish to consider the estimation of all estimable parametric functions of $\boldsymbol{\beta}_{1}$ under $\mathscr{F}$, then it is equivalent to consider $\mathbf{M}_{2} \mathbf{X}_{1} \boldsymbol{\beta}_{1}$. In other words, the reason to concentrate on estimating $\boldsymbol{\theta}_{1}=\mathbf{M}_{2} \mathbf{X}_{1} \boldsymbol{\beta}_{1}$ is that the properties obtained are valid for all parametric functions of the type $\mathbf{K}_{1} \boldsymbol{\beta}_{1}$ that are estimable under the partitioned model $\mathscr{F}$. 
Clearly if $\mathbf{K}_{1} \boldsymbol{\beta}_{1}$ is estimable under $\mathscr{F}$ then it is estimable under $\mathscr{M}$.

It is well known that $\boldsymbol{\mu}_{1}=\mathbf{X}_{1} \boldsymbol{\beta}_{1}$ is estimable in $\mathscr{F}$ if and only if

$$
\mathscr{C}\left(\mathbf{X}_{1}\right) \cap \mathscr{C}\left(\mathbf{X}_{2}\right)=\{\mathbf{0}\} \text {. }
$$

This follows from the requirement $\mathscr{C}\left(\mathbf{X}_{1}^{\prime}\right) \subseteq \mathscr{C}\left(\mathbf{X}_{1}^{\prime} \mathbf{M}_{2}\right)$, i.e., $\mathscr{C}\left(\mathbf{X}_{1}^{\prime}\right)=$ $\mathscr{C}\left(\mathbf{X}_{1}^{\prime} \mathbf{M}_{2}\right)$, which holds if and only if (1) holds.

For Lemma 1.1, characterizing the BLUE, see, e.g., Rao [20, p. 282], and the BLUP, see, e.g., Christensen [1, p. 294], and [12, p. 1015]. For further references, see Haslett et al. 33, 4]. For the general reviews of the BLUPproperties, see, e.g., Tian [23, 24].

Lemma 1.1. Consider the models $\mathscr{F}$ and $\mathscr{M}$, and denote $\boldsymbol{\Sigma}=\mathbf{X}_{2} \mathbf{D} \mathbf{X}_{2}^{\prime}+$ V. Then the following statements hold.

(a) $\mathbf{A}_{1} \mathbf{y}$ is the BLUE for estimable $\mathbf{K} \boldsymbol{\beta}$ under $\mathscr{F}$ if and only if

$$
\mathbf{A}_{1}(\mathbf{X}: \mathbf{V M})=(\mathbf{K}: \mathbf{0}) \text {, i.e., } \mathbf{A}_{1} \in\left\{\mathbf{P}_{\mathbf{K} \boldsymbol{\beta} \mid \mathscr{F}}\right\} \text {. }
$$

(b) $\mathbf{A}_{2} \mathbf{y}$ is the BLUE for estimable $\mathbf{K}_{1} \boldsymbol{\beta}_{1}$ under $\mathscr{M}$ if and only if

$$
\mathbf{A}_{2}\left(\mathbf{X}_{1}: \mathbf{\Sigma} \mathbf{M}_{1}\right)=\left(\mathbf{K}_{1}: \mathbf{0}\right) \text {, i.e., } \mathbf{A}_{2} \in\left\{\mathbf{P}_{\mathbf{K}_{1} \boldsymbol{\beta}_{1} \mid \mathscr{M}}\right\} \text {. }
$$

(c) $\mathbf{A}_{3} \mathbf{y}$ is the BLUP for $\mathbf{J u}$ under $\mathscr{M}$ if and only if

$$
\mathbf{A}_{3}\left(\mathbf{X}_{1}: \mathbf{\Sigma} \mathbf{M}_{1}\right)=\left(\mathbf{0}: \mathbf{J D} \mathbf{J}^{\prime} \mathbf{M}_{1}\right) \text {, i.e., } \mathbf{A}_{3} \in\left\{\mathbf{P}_{\mathbf{J u} \mid \mathscr{M}}\right\} \text {. }
$$

(d) $\mathbf{A}_{4} \mathbf{y}$ is the BLUP for $\mathbf{g}=\mathbf{K}_{1} \boldsymbol{\beta}_{1}+\mathbf{J u}$ under $\mathscr{M}$ if and only if

$$
\mathbf{A}_{4}\left(\mathbf{X}_{1}: \mathbf{\Sigma} \mathbf{M}_{1}\right)=\left(\mathbf{K}_{1}: \mathbf{J D J} \mathbf{J}^{\prime} \mathbf{M}_{1}\right) \text {, i.e., } \mathbf{A}_{4} \in\left\{\mathbf{P}_{\mathbf{g} \mid \mathscr{M}}\right\} \text {. }
$$

Remark 1.1. Notice the difference between the notations like

$$
\mathbf{P}_{\mathbf{A}}=\mathbf{A A}^{+}, \quad\left\{\mathbf{P}_{\mathbf{K}_{1} \boldsymbol{\beta}_{1} \mid \mathscr{M}}\right\} .
$$

Above $\mathbf{P}_{\mathbf{A}}$ is the (unique) orthogonal projector onto $\mathscr{C}(\mathbf{A})$, while $\left\{\mathbf{P}_{\left.\mathbf{K}_{1} \boldsymbol{\beta}_{1} \mid \mathscr{M}\right\}}\right.$ is a set of matrices $\mathbf{A}_{2}$ satisfying $\mathbf{A}_{2}\left(\mathbf{X}_{1}: \mathbf{\Sigma} \mathbf{M}_{1}\right)=\left(\mathbf{K}_{1}: \mathbf{0}\right)$.

If $\mathbf{A}_{2} \in\left\{\mathbf{P}_{\mathbf{K}_{1} \boldsymbol{\beta}_{1} \mid \mathscr{M}}\right\}$ and $\mathbf{A}_{3} \in\left\{\mathbf{P}_{\mathbf{J u} \mid \mathscr{M}}\right\}$, i.e.,

$$
\left(\begin{array}{l}
\mathbf{A}_{2} \\
\mathbf{A}_{3}
\end{array}\right)\left(\mathbf{X}_{1}: \mathbf{\Sigma} \mathbf{M}_{1}\right)=\left(\begin{array}{cc}
\mathbf{K}_{1} & \mathbf{0} \\
\mathbf{0} & \mathbf{J D J}^{\prime} \mathbf{M}_{1}
\end{array}\right)
$$

then premultiplying $(2)$ by $\left(\mathbf{I}_{q}: \mathbf{I}_{q}\right)$ we immediately see that

$$
\mathbf{A}_{2}+\mathbf{A}_{3} \in\left\{\mathbf{P}_{\mathbf{K}_{1} \boldsymbol{\beta}_{1}+\mathbf{J u} \mid \mathscr{M}}\right\}
$$

i.e., under $\mathscr{M}$ we have

$$
\operatorname{BLUP}\left(\mathbf{K}_{1} \boldsymbol{\beta}_{1}+\mathbf{J u}\right)=\operatorname{BLUE}\left(\mathbf{K}_{1} \boldsymbol{\beta}_{1}\right)+\operatorname{BLUP}(\mathbf{J u}) .
$$

It is well known, see, e.g., Rao [20], that

$$
\mathbf{G}=\mathbf{X}\left(\mathbf{X}^{\prime} \mathbf{W}^{-} \mathbf{X}\right)^{-} \mathbf{X}^{\prime} \mathbf{W}^{+},
$$


where

$$
\mathbf{W}=\mathbf{X}_{1} \mathbf{X}_{1}^{\prime}+\mathbf{X}_{2} \mathbf{X}_{2}^{\prime}+\mathbf{V}=\mathbf{X} \mathbf{X}^{\prime}+\mathbf{V}
$$

is one solution to the equation $\mathbf{A}(\mathbf{X}: \mathbf{V M})=(\mathbf{X}: \mathbf{0})$; recall that $\boldsymbol{\mu}=\mathbf{X} \boldsymbol{\beta}$ is always estimable in $\mathscr{F}$. The matrix $\mathbf{G}$ is unique for the choice of generalized inverses marked as "-" but to obtain uniqueness for $\mathbf{G}$ (which somewhat simplifies our considerations) we have to choose the Moore-Penrose inverse $\mathbf{W}^{+}$in the end of the expression (4).

Below are some solutions to equations appearing in Lemma 1.1 (for references, see, e.g. [19, Ch. 10]):

$$
\begin{array}{rlrl}
\mathbf{G}_{\boldsymbol{\mu}_{1} \mid \mathscr{F}} & =\mathbf{X}_{1}\left(\mathbf{X}_{1}^{\prime} \dot{\mathbf{M}}_{2} \mathbf{X}_{1}\right)^{-} \mathbf{X}_{1}^{\prime} \dot{\mathbf{M}}_{2} & & \in\left\{\mathbf{P}_{\boldsymbol{\mu}_{1} \mid \mathscr{F}}\right\}, \\
\mathbf{G}_{\boldsymbol{\theta}_{1} \mid \mathscr{F}} & =\mathbf{M}_{2} \mathbf{G}_{\boldsymbol{\mu}_{1} \mid \mathscr{F}} & & \in\left\{\mathbf{P}_{\boldsymbol{\theta}_{1} \mid \mathscr{F}}\right\}, \\
\mathbf{G}_{\boldsymbol{\theta}_{2} \mid \mathscr{F}} & =\mathbf{M}_{1} \mathbf{X}_{2}\left(\mathbf{X}_{2}^{\prime} \dot{\mathbf{M}}_{1} \mathbf{X}_{2}\right)^{-} \mathbf{X}_{2}^{\prime} \dot{\mathbf{M}}_{1} & & \in\left\{\mathbf{P}_{\boldsymbol{\theta}_{2} \mid \mathscr{F}}\right\}, \\
\mathbf{G}_{\boldsymbol{\mu}_{1} \mid \mathscr{M}} & =\mathbf{X}_{1}\left(\mathbf{X}_{1}^{\prime} \mathbf{W}_{m}^{-} \mathbf{X}_{1}\right)^{-} \mathbf{X}_{1}^{\prime} \mathbf{W}_{m}^{+} & & \in\left\{\mathbf{P}_{\left.\boldsymbol{\mu}_{1} \mid \mathscr{M}\right\},},\right. \\
\mathbf{G}_{\boldsymbol{\theta}_{1} \mid \mathscr{M}} & =\mathbf{M}_{2} \mathbf{G}_{\boldsymbol{\mu}_{1} \mid \mathscr{M}} & \in\left\{\mathbf{P}_{\boldsymbol{\theta}_{1} \mid \mathscr{M}}\right\}, \\
\mathbf{G}_{\mathbf{X}_{2} \mathbf{u} \mid \mathscr{M}} & =\mathbf{X}_{2} \mathbf{D} \mathbf{X}_{2}^{\prime} \mathbf{M}_{1}\left(\mathbf{M}_{1} \boldsymbol{\Sigma} \mathbf{M}_{1}\right)^{+} \mathbf{M}_{1} & \in\left\{\mathbf{P}_{\mathbf{X}_{2} \mathbf{u} \mid \mathscr{M}}\right\}, \\
\mathbf{G}_{\mathbf{M}_{1} \mathbf{X}_{2} \mathbf{u} \mid \mathscr{M}} & =\mathbf{M}_{1} \mathbf{G}_{\mathbf{X}_{2} \mathbf{u} \mid \mathscr{M}} & \in\left\{\mathbf{P}_{\left.\mathbf{M}_{1} \mathbf{X}_{2} \mathbf{u} \mid \mathscr{M}\right\},}\right.
\end{array}
$$

where $\boldsymbol{\theta}_{2}=\mathbf{M}_{1} \mathbf{X}_{2} \boldsymbol{\beta}_{2}$ and

$$
\mathbf{W}_{m}=\mathbf{X}_{1} \mathbf{X}_{1}^{\prime}+\boldsymbol{\Sigma}=\mathbf{X}_{1} \mathbf{X}_{1}^{\prime}+\mathbf{X}_{2} \mathbf{D} \mathbf{X}_{2}^{\prime}+\mathbf{V} \text {. }
$$

The matrices $\dot{\mathbf{M}}_{1}$ and $\dot{\mathbf{M}}_{2}$ are defined as

$$
\dot{\mathbf{M}}_{1}=\mathbf{M}_{1}\left(\mathbf{M}_{1} \mathbf{W} \mathbf{M}_{1}\right)^{+} \mathbf{M}_{1}, \quad \dot{\mathbf{M}}_{2}=\mathbf{M}_{2}\left(\mathbf{M}_{2} \mathbf{W} \mathbf{M}_{2}\right)^{+} \mathbf{M}_{2} .
$$

Moreover, see, e.g., [19, Ch. 15],

$$
\dot{\mathbf{M}}_{2}=\mathbf{M}_{2}\left(\mathbf{M}_{2} \mathbf{W} \mathbf{M}_{2}\right)^{+} \mathbf{M}_{2}=\mathbf{M}_{2}\left(\mathbf{M}_{2} \mathbf{W} \mathbf{M}_{2}\right)^{+}=\left(\mathbf{M}_{2} \mathbf{W} \mathbf{M}_{2}\right)^{+} .
$$

Obviously, denoting $\mathbf{W}_{1}=\mathbf{X}_{1} \mathbf{X}_{1}^{\prime}+\mathbf{V}$, we have

$$
\mathbf{M}_{2} \mathbf{W}=\mathbf{M}_{2} \mathbf{W}_{1}=\mathbf{M}_{2} \mathbf{W}_{m}, \quad \mathbf{M}_{1} \mathbf{W}_{m}=\mathbf{M}_{1} \boldsymbol{\Sigma} .
$$

It is not necessary to choose $\mathbf{W}$ and $\mathbf{W}_{m}$ as in (5) and in (6). For example, $\mathbf{W}$ could be replaced with $\mathbf{W}_{*}=\mathbf{X} \mathbf{U U}^{\prime} \mathbf{X}^{\prime}+\mathbf{V}$ such that $\mathscr{C}\left(\mathbf{W}_{*}\right)=\mathscr{C}(\mathbf{X}: \mathbf{V})$; see, e.g., [19, Sec. 12.3].

The solutions to equations in Lemma 1.1 dealing with $\mathscr{F}$ are unique if and only if $\mathscr{C}(\mathbf{W})=\mathbb{R}^{n}$ while those dealing with $\mathscr{M}$ are unique if and only if $\mathscr{C}\left(\mathbf{W}_{m}\right)=\mathbb{R}^{n}$. The general solution for $\mathbf{A}$ in

$$
\mathbf{A}\left(\mathbf{X}_{1}: \mathbf{X}_{2}: \mathbf{V M}\right)=\left(\mathbf{M}_{2} \mathbf{X}_{1}: \mathbf{0}: \mathbf{0}\right)
$$

can be expressed, e.g., as

$$
\mathbf{A}_{0}=\mathbf{G}_{\boldsymbol{\theta}_{1} \mid \mathscr{F}}+\mathbf{E} \mathbf{Q}_{\mathbf{W}}=\mathbf{M}_{2} \mathbf{X}_{1}\left(\mathbf{X}_{1}^{\prime} \dot{\mathbf{M}}_{2} \mathbf{X}_{1}\right)^{-} \mathbf{X}_{1}^{\prime} \dot{\mathbf{M}}_{2}+\mathbf{E} \mathbf{Q}_{\mathbf{W}},
$$

where $\mathbf{E} \in \mathbb{R}^{n \times n}$ is free to vary. By the consistency of the model $\mathscr{F}$ it is meant that $\mathbf{y}$ lies in $\mathscr{C}(\mathbf{W})$ with probability 1 . Thus under the consistent 
model $\mathscr{F}$ the vector $\mathbf{A}_{0} \mathbf{y}$ itself is unique once $\mathbf{y}$ has been observed. The consistency in $\mathscr{M}$ means that $\mathbf{y}$ belongs to $\mathscr{C}\left(\mathbf{W}_{m}\right)$. Notice that

$$
\mathscr{C}\left(\mathbf{W}_{m}\right)=\mathscr{C}\left(\mathbf{X}_{1}: \mathbf{X}_{2} \mathbf{D}: \mathbf{V}\right) \subseteq \mathscr{C}\left(\mathbf{X}_{1}: \mathbf{X}_{2}: \mathbf{V}\right)=\mathscr{C}(\mathbf{W}),
$$

with equality holding if and only if $\mathscr{C}\left(\mathbf{X}_{2}\right) \subseteq \mathscr{C}\left(\mathbf{W}_{m}\right)=\mathscr{C}\left(\mathbf{X}_{1}: \mathbf{\Sigma} \mathbf{M}_{1}\right)$.

In the consistent linear model $\mathscr{F}$, the estimators $\mathbf{A y}$ and $\mathbf{B y}$ are said to be equal (with probability 1 ) if

$$
\mathbf{A y}=\mathbf{B y} \text { for all } \mathbf{y} \in \mathscr{C}(\mathbf{X}: \mathbf{V})=\mathscr{C}(\mathbf{X}: \mathbf{V M})=\mathscr{C}(\mathbf{X}) \oplus \mathscr{C}(\mathbf{V M}),
$$

where $\oplus$ refers to the direct sum. In (7) we are dealing with the "statistical" equality of the estimators $\mathbf{A y}$ and $\mathbf{B y}$. In (7) $\mathbf{y}$ refers to a vector in $\mathbb{R}^{n}$, while in the notation $\operatorname{cov}(\mathbf{A y})$ we understand $\mathbf{y}$ as a random vector. We may consider, for example, the equation

$$
\mathbf{G}_{\boldsymbol{\theta}_{1} \mid \mathscr{F}} \mathbf{y}=\mathbf{G}_{\boldsymbol{\theta}_{1} \mid \mathscr{M}} \mathbf{y}
$$

but now we immediately observe some problems in defining the space where $\mathbf{y}$ is varying in (8). We can write, for example,

$$
\mathbf{G}_{\boldsymbol{\theta}_{1} \mid \mathscr{F}} \mathbf{y}=\operatorname{BLUE}\left(\boldsymbol{\theta}_{1} \mid \mathscr{F}\right), \quad \mathbf{G}_{\boldsymbol{\theta}_{1} \mid \mathscr{M}} \mathbf{y}=\operatorname{BLUE}\left(\boldsymbol{\theta}_{1} \mid \mathscr{M}\right),
$$

which are short notations for phrases like " $\mathbf{G}_{\boldsymbol{\theta}_{1} \mid \mathscr{M}} \mathbf{y}$ is the BLUE for $\boldsymbol{\theta}_{1}$ under $\mathscr{F} "$ etc. However, writing the equalities like

$$
\operatorname{BLUE}\left(\boldsymbol{\mu}_{1} \mid \mathscr{F}\right)=\operatorname{BLUE}\left(\boldsymbol{\mu}_{1} \mid \mathscr{M}\right),
$$

may cause problems when the representations are not unique.

Isotalo et al. 11] found conditions under which an arbitrary representation of the BLUE of $\boldsymbol{\theta}_{1}=\mathbf{M}_{2} \mathbf{X}_{1} \boldsymbol{\beta}_{1}$ under the fixed model $\mathscr{F}$ remains the BLUE for $\boldsymbol{\theta}_{1}$ under the mixed model $\mathscr{M}$. This kind of property can be denoted shortly as

$$
\left\{\operatorname{BLUE}\left(\boldsymbol{\theta}_{1} \mid \mathscr{F}\right)\right\} \subseteq\left\{\operatorname{BLUE}\left(\boldsymbol{\theta}_{1} \mid \mathscr{M}\right)\right\},
$$

or, equivalently as $\left\{\mathbf{P}_{\boldsymbol{\theta}_{1} \mid \mathscr{F}}\right\} \subseteq\left\{\mathbf{P}_{\boldsymbol{\theta}_{1} \mid \mathscr{M}}\right\}$, where the sets $\left\{\mathbf{P}_{\boldsymbol{\theta}_{1} \mid \mathscr{F}}\right\}$ and $\left\{\mathbf{P}_{\boldsymbol{\theta}_{1} \mid \mathscr{M}}\right\}$ are defined as in Lemma 1.1

$$
\begin{aligned}
& \mathbf{A} \in\left\{\mathbf{P}_{\boldsymbol{\theta}_{1} \mid \mathscr{F}}\right\} \Longleftrightarrow \mathbf{A}\left(\mathbf{X}_{1}: \mathbf{X}_{2}: \mathbf{V M}\right)=\left(\mathbf{M}_{2} \mathbf{X}_{1}: \mathbf{0}: \mathbf{0}\right), \\
& \mathbf{B} \in\left\{\mathbf{P}_{\boldsymbol{\theta}_{1} \mid \mathscr{M}}\right\} \Longleftrightarrow \mathbf{B}\left(\mathbf{X}_{1}: \mathbf{\Sigma} \mathbf{M}_{1}\right)=\left(\mathbf{M}_{2} \mathbf{X}_{1}: \mathbf{0}\right) .
\end{aligned}
$$

In this paper we generalize the results of Isotalo et al. [11] by considering the following relations:

$$
\begin{array}{rlll}
\operatorname{BLUE}\left(\mathbf{M}_{2} \mathbf{X}_{1} \boldsymbol{\beta}_{1} \mid \mathscr{F}\right) & \text { vs } & \operatorname{BLUP}\left(\mathbf{M}_{2} \mathbf{X}_{1} \boldsymbol{\beta}_{1}+\mathbf{X}_{2} \mathbf{u} \mid \mathscr{M}\right), \\
\operatorname{BLUE}\left(\mathbf{M}_{2} \mathbf{X}_{2} \boldsymbol{\beta}_{2} \mid \mathscr{F}\right) & \text { vs } & \operatorname{BLUP}\left(\mathbf{M}_{2} \mathbf{X}_{2} \mathbf{u} \mid \mathscr{M}\right), \\
\operatorname{BLUE}(\mathbf{X} \boldsymbol{\beta} \mid \mathscr{F}) & \text { vs } & \operatorname{BLUP}\left(\mathbf{X}_{1} \boldsymbol{\beta}_{1}+\mathbf{X}_{2} \mathbf{u} \mid \mathscr{M}\right) .
\end{array}
$$

The case of two linear fixed models $\mathscr{B}_{i}=\left\{\mathbf{y}, \mathbf{X} \boldsymbol{\beta}, \mathbf{V}_{i}\right\}, i=1,2$, with different covariance matrices is extensively studied by Mitra and Moore [18]. Haslett et al. [7] provide a review of conditions under which BLUEs/BLUPs 
in one linear mixed model are also BLUE/BLUPs in another (with possibly different design matrices and covariance structures).

We end this section with a useful lemma.

Lemma 1.2. Using the earlier notation, the following statements hold:

(a) $\mathbf{M}=\mathbf{I}_{n}-\mathbf{P}_{\left(\mathbf{X}_{1}: \mathbf{X}_{2}\right)}=\mathbf{I}_{n}-\left(\mathbf{P}_{\mathbf{X}_{2}}+\mathbf{P}_{\mathbf{M}_{2} \mathbf{X}_{1}}\right)=\mathbf{M}_{2} \mathbf{Q}_{\mathbf{M}_{2} \mathbf{X}_{1}}=\mathbf{Q}_{\mathbf{M}_{2} \mathbf{X}_{1}} \mathbf{M}_{2}$,

(b) $\mathrm{r}\left(\mathbf{M}_{2} \mathbf{X}_{1}\right)=\mathrm{r}\left(\mathbf{X}_{1}\right)-\operatorname{dim} \mathscr{C}\left(\mathbf{X}_{1}\right) \cap \mathscr{C}\left(\mathbf{X}_{2}\right)$

(c) $\mathrm{r}(\mathbf{A B})=\mathrm{r}(\mathbf{A})-\operatorname{dim} \mathscr{C}\left(\mathbf{A}^{\prime}\right) \cap \mathscr{C}(\mathbf{B})^{\perp}$,

(d) $\mathscr{C}\left(\mathbf{W}^{+} \mathbf{X}\right)^{\perp}=\mathscr{C}\left(\mathbf{W M}: \mathbf{Q}_{\mathbf{W}}\right)=\mathscr{C}\left(\mathbf{V M}: \mathbf{Q}_{\mathbf{W}}\right)$

(e) $\mathscr{C}\left(\mathbf{X}_{2}: \mathbf{\Sigma} \mathbf{M}\right)=\mathscr{C}\left[\mathbf{M}_{2}\left(\mathbf{M}_{2} \mathbf{W} \mathbf{M}_{2}\right)^{+} \mathbf{M}_{2} \mathbf{X}_{1}: \mathbf{Q} \mathbf{W}\right]^{\perp}$,

(f) $\mathscr{C}\left[\mathbf{A}\left(\mathbf{A}^{\prime} \mathbf{B}^{\perp}\right)^{\perp}\right]=\mathscr{C}(\mathbf{A}) \cap \mathscr{C}(\mathbf{B})$.

For part (b) and (c), see, e.g., [17, Cor. 6.2]. For (d), see, e.g., [16, Lemma 4] and [20, Sec. 2]. For (e), see [11, Lemma, p. 72], and for (f), see [21, Compl. 7, p. 118].

\section{Equality between the BLUEs}

Isotalo et al. [11, Sec. 2] proved the following result:

Theorem 2.1. The following statements hold.

(a) An arbitrary BLUE for $\boldsymbol{\theta}_{1}=\mathbf{M}_{2} \mathbf{X}_{1} \boldsymbol{\beta}_{1}$ under $\mathscr{F}$ provides also the BLUE for $\boldsymbol{\theta}_{1}$ under the mixed model $\mathscr{M}$, i.e.,

$$
\left\{\operatorname{BLUE}\left(\boldsymbol{\theta}_{1} \mid \mathscr{F}\right)\right\} \subseteq\left\{\operatorname{BLUE}\left(\boldsymbol{\theta}_{1} \mid \mathscr{M}\right)\right\},
$$

i.e., $\left\{\mathbf{P}_{\boldsymbol{\theta}_{1} \mid \mathscr{F}}\right\} \subseteq\left\{\mathbf{P}_{\boldsymbol{\theta}_{1} \mid \mathscr{M}}\right\}$, holds if and only if

$$
\mathscr{C}\left(\mathbf{\Sigma M}_{1}\right) \subseteq \mathscr{C}\left(\mathbf{X}_{2}: \mathbf{V M}\right) .
$$

(b) The reverse relation $\left\{\operatorname{BLUE}\left(\boldsymbol{\theta}_{1} \mid \mathscr{M}\right)\right\} \subseteq\left\{\operatorname{BLUE}\left(\boldsymbol{\theta}_{1} \mid \mathscr{F}\right)\right\}$, i.e., $\left\{\mathbf{P}_{\boldsymbol{\theta}_{1} \mid \mathscr{M}}\right\} \subseteq\left\{\mathbf{P}_{\boldsymbol{\theta}_{1} \mid \mathscr{F}}\right\}$, holds if and only if

$$
\mathscr{C}\left(\mathbf{X}_{2}: \mathbf{V M}\right) \subseteq \mathscr{C}\left(\mathbf{R}: \mathbf{\Sigma} \mathbf{M}_{1}\right) \text {, i.e., } \mathscr{C}\left(\mathbf{X}_{2}\right) \subseteq \mathscr{C}\left(\mathbf{R}: \mathbf{\Sigma} \mathbf{M}_{1}\right),
$$

where the matrix $\mathbf{R}$ has property $\mathscr{C}(\mathbf{R})=\mathscr{C}\left(\mathbf{X}_{1}\right) \cap \mathscr{C}\left(\mathbf{X}_{2}\right)$.

Actually, the matrix $\mathbf{R}$ in (11) was erroneously missing in [11. Notice that the equivalence of the two inclusions in 111) follows from $\mathscr{C}(\mathbf{V M})=$ $\mathscr{C}(\boldsymbol{\Sigma} \mathbf{M}) \subseteq \mathscr{C}\left(\boldsymbol{\Sigma} \mathbf{M}_{1}\right)$, which is based on

$$
\mathscr{C}(\mathbf{M})=\mathscr{C}\left(\mathbf{M}_{1} \mathbf{Q}_{\mathbf{M}_{1} \mathbf{X}_{2}}\right) \subseteq \mathscr{C}\left(\mathbf{M}_{1}\right) .
$$

The inclusion $(10)$ is obviously equivalent to $\mathscr{C}\left(\mathbf{R}: \mathbf{\Sigma} \mathbf{M}_{1}\right) \subseteq \mathscr{C}\left(\mathbf{X}_{2}: \mathbf{V M}\right)$ and thereby $\left\{\mathbf{P}_{\boldsymbol{\theta}_{1} \mid \mathscr{M}}\right\}=\left\{\mathbf{P}_{\boldsymbol{\theta}_{1} \mid \mathscr{F}}\right\}$ holds if and only if

$$
\mathscr{C}\left(\mathbf{R}: \mathbf{\Sigma} \mathbf{M}_{1}\right)=\mathscr{C}\left(\mathbf{X}_{2}: \mathbf{V M}\right) .
$$

Moreover, it is interesting to observe that (10) is equivalent to

$$
\mathscr{C}\left(\mathbf{V M}_{1}\right) \subseteq \mathscr{C}\left(\mathbf{X}_{2}: \mathbf{V M}\right) \text {. }
$$


Namely, writing $\mathbf{P}_{\left(\mathbf{X}_{2}: \mathbf{V M}\right)}=\mathbf{P}_{\mathbf{X}_{2}}+\mathbf{P}_{\mathbf{M}_{2} \mathbf{V M}}$, it is easy to confirm that

$$
\mathbf{P}_{\left(\mathbf{X}_{2}: \mathrm{VM}\right)} \mathbf{V M}_{1}=\mathbf{V M}_{1} \Longleftrightarrow \mathbf{P}_{\left(\mathrm{X}_{2}: \mathrm{VM}\right)} \boldsymbol{\Sigma} \mathbf{M}_{1}=\boldsymbol{\Sigma} \mathbf{M}_{1}
$$

If $\boldsymbol{\mu}_{1}=\mathbf{X}_{1} \boldsymbol{\beta}_{1}$ is estimable under $\mathscr{F}$, i.e., $\mathscr{C}\left(\mathbf{X}_{1}\right) \cap \mathscr{C}\left(\mathbf{X}_{2}\right)=\{\mathbf{0}\}$, we immdiately observe that (11) simplifies into $\mathscr{C}\left(\mathbf{X}_{2}\right) \subseteq \mathscr{C}\left(\boldsymbol{\Sigma} \mathbf{M}_{1}\right)$. Moreover, we can obtain the following corollary.

Corollary 2.1. Let $\boldsymbol{\mu}_{1}=\mathbf{X}_{1} \boldsymbol{\beta}_{1}$ is estimable under $\mathscr{F}$. Then the following statements are equivalent:

(a) $\left\{\operatorname{BLUE}\left(\boldsymbol{\mu}_{1} \mid \mathscr{M}\right)\right\} \subseteq\left\{\operatorname{BLUE}\left(\boldsymbol{\mu}_{1} \mid \mathscr{F}\right)\right\}$,

(b) $\left\{\operatorname{BLUE}\left(\boldsymbol{\mu}_{1} \mid \mathscr{M}\right)\right\}=\left\{\operatorname{BLUE}\left(\boldsymbol{\mu}_{1} \mid \mathscr{F}\right)\right\}$,

(c) $\mathscr{C}\left(\mathbf{X}_{2}: \mathbf{V M}\right) \subseteq \mathscr{C}\left(\boldsymbol{\Sigma} \mathbf{M}_{1}\right)$,

(d) $\mathscr{C}\left(\mathbf{X}_{2}: \mathbf{V M}\right)=\mathscr{C}\left(\boldsymbol{\Sigma} \mathbf{M}_{1}\right)$,

(e) $\mathscr{C}\left(\mathbf{X}_{2}\right) \subseteq \mathscr{C}\left(\boldsymbol{\Sigma} \mathbf{M}_{1}\right)$.

Proof. The equivalence of (a), (c) and (e) follows from Theorem 2.1. Assuming the disjointness $\mathscr{C}\left(\mathbf{X}_{1}\right) \cap \mathscr{C}\left(\mathbf{X}_{2}\right)=\{\mathbf{0}\}$, we observe, using (c) of Lemma 1.2 , that

$$
\begin{aligned}
\mathrm{r}\left(\mathbf{X}_{2}: \boldsymbol{\Sigma} \mathbf{M}\right) & =\mathrm{r}\left(\mathbf{X}_{2}\right)+\mathrm{r}(\boldsymbol{\Sigma} \mathbf{M})=\mathrm{r}\left(\mathbf{X}_{2}\right)+\mathrm{r}\left(\boldsymbol{\Sigma}_{1} \mathbf{Q}_{\left.\mathbf{M}_{1} \mathbf{X}_{2}\right)}\right. \\
& =\mathrm{r}\left(\mathbf{X}_{2}\right)+\mathrm{r}\left(\boldsymbol{\Sigma} \mathbf{M}_{1}\right)-\operatorname{dim} \mathscr{C}\left(\mathbf{M}_{1} \boldsymbol{\Sigma}\right) \cap \mathscr{C}\left(\mathbf{M}_{1} \mathbf{X}_{2}\right) \\
& \geq \mathrm{r}\left(\mathbf{X}_{2}\right)+\mathrm{r}\left(\boldsymbol{\Sigma} \mathbf{M}_{1}\right)-\mathrm{r}\left(\mathbf{M}_{1} \mathbf{X}_{2}\right)=\mathrm{r}\left(\boldsymbol{\Sigma} \mathbf{M}_{1}\right) .
\end{aligned}
$$

Thereby, if (c) holds, then (12) implies that necessarily (d) holds, which further is equivalent to (b).

Remark 2.1. Isotalo et al. [11, p. 72] considered also the condition under which there exists at least one representation of the BLUE of $\boldsymbol{\theta}_{1}$ under $\mathscr{F}$ which is also BLUE of $\boldsymbol{\theta}_{1}$ under $\mathscr{M}$. This means that there exists a matrix A such that $\mathbf{A} \in\left\{\mathbf{P}_{\boldsymbol{\theta}_{1} \mid \mathscr{F}}\right\} \cap\left\{\mathbf{P}_{\boldsymbol{\theta}_{1} \mid \mathscr{M}}\right\}$, i.e., $\mathbf{A}$ satisfies the equation

$$
\mathbf{A}\left(\mathbf{X}_{1}: \mathbf{X}_{2}: \boldsymbol{\Sigma} \mathbf{M}_{1}: \boldsymbol{\Sigma M}\right)=\left(\mathbf{M}_{2} \mathbf{X}_{1}: \mathbf{0}: \mathbf{0}: \mathbf{0}\right) \text {. }
$$

It is clear that $\mathbf{A} \mathbf{\Sigma} \mathbf{M}_{1}=\mathbf{0}$ implies $\mathbf{A} \mathbf{\Sigma} \mathbf{M}=\mathbf{0}$ and so $(13)$ is equivalent to

$$
\mathbf{A}\left(\mathbf{X}_{1}: \mathbf{X}_{2}: \mathbf{\Sigma} \mathbf{M}_{1}\right)=\left(\mathbf{M}_{2} \mathbf{X}_{1}: \mathbf{0}: \mathbf{0}\right) \text {. }
$$

Now (14) has a solution for $\mathbf{A}$ if and only if

$$
\mathscr{N}\left(\mathbf{X}_{1}: \mathbf{X}_{2}: \mathbf{\Sigma} \mathbf{M}_{1}\right) \subseteq \mathscr{N}\left(\mathbf{M}_{2} \mathbf{X}_{1}: \mathbf{0}: \mathbf{0}\right),
$$

where $\mathscr{N}(\cdot)$ refers to the nullspace. The corresponding conditions for further relations appearing in this article can be introduced (we will omit them).

It is interesting to consider the "statistical" equality

$$
\mathbf{G}_{\boldsymbol{\theta}_{1} \mid \mathscr{F}} \mathbf{y}=\mathbf{G}_{\boldsymbol{\theta}_{1} \mid \mathscr{M}} \mathbf{y}
$$

in deeper details. In particular we can consider two cases:

$$
\mathbf{y} \in \mathscr{C}(\mathbf{W})=\mathscr{C}\left(\mathbf{X}_{1}: \mathbf{X}_{2}: \mathbf{V}\right), \quad \mathbf{y} \in \mathscr{C}\left(\mathbf{W}_{m}\right)=\mathscr{C}\left(\mathbf{X}_{1}: \mathbf{X}_{2} \mathbf{D}: \mathbf{V}\right)
$$


Recall that in the fixed model $\mathscr{F}$ the "permissible observation space" for the response variable $\mathbf{y}$ is $\mathscr{C}(\mathbf{W})$ while in the mixed model $\mathscr{M}$ it is $\mathscr{C}\left(\mathbf{W}_{m}\right)$. Now the following corollary is straightforward to confirm.

Corollary 2.2. Consider the models $\mathscr{F}$ and $\mathscr{M}$.

(a) The following statements are equivalent:

(i) $\mathbf{G}_{\boldsymbol{\theta}_{1} \mid \mathscr{M}} \mathbf{y}=\mathbf{G}_{\boldsymbol{\theta}_{1} \mid \mathscr{F}} \mathbf{y}$ for all $\mathbf{y} \in \mathscr{C}(\mathbf{W})=\mathscr{C}\left(\mathbf{X}_{1}: \mathbf{X}_{2}: \mathbf{V}\right)$,

(ii) $\mathbf{G}_{\boldsymbol{\theta}_{1} \mid \mathscr{M}}\left(\mathbf{X}_{1}: \mathbf{X}_{2}: \mathbf{V M}\right)=\left(\mathbf{M}_{2} \mathbf{X}_{1}: \mathbf{0}: \mathbf{0}\right)$,

(iii) $\mathbf{G}_{\boldsymbol{\theta}_{1} \mid \mathscr{M}} \in\left\{\mathbf{P}_{\boldsymbol{\theta}_{1} \mid \mathscr{F}}\right\}$, i.e., $\mathbf{G}_{\boldsymbol{\theta}_{1} \mid \mathscr{M}} \mathbf{y}=\operatorname{BLUE}\left(\boldsymbol{\theta}_{1} \mid \mathscr{F}\right)$.

(b) The following statements are equivalent:

(i) $\left(\mathbf{G}_{\boldsymbol{\theta}_{1} \mid \mathscr{M}}+\mathbf{E Q}_{\mathbf{W}_{m}}\right) \mathbf{y}=\mathbf{G}_{\boldsymbol{\theta}_{1} \mid \mathscr{F}} \mathbf{y}$ for all $\mathbf{y} \in \mathscr{C}(\mathbf{W})$ and for all $\mathbf{E}$,

(ii) $\left(\mathbf{G}_{\boldsymbol{\theta}_{1} \mid \mathscr{M}}+\mathbf{E Q}_{\mathbf{W}_{m}}\right)\left(\mathbf{X}_{1}: \mathbf{X}_{2}: \mathbf{V M}\right)=\left(\mathbf{M}_{2} \mathbf{X}_{1}: \mathbf{0}: \mathbf{0}\right)$ for all $\mathbf{E}$,

(iii) $\left\{\operatorname{BLUE}\left(\boldsymbol{\theta}_{1} \mid \mathscr{M}\right)\right\} \subseteq\left\{\operatorname{BLUE}\left(\boldsymbol{\theta}_{1} \mid \mathscr{F}\right)\right\}$.

(c) The following statements are equivalent:

(i) $\mathbf{G}_{\boldsymbol{\theta}_{1} \mid \mathscr{F}} \mathbf{y}=\mathbf{G}_{\boldsymbol{\theta}_{1} \mid \mathscr{M}} \mathbf{y}$ for all $\mathbf{y} \in \mathscr{C}\left(\mathbf{W}_{m}\right)=\mathscr{C}\left(\mathbf{X}_{1}: \mathbf{\Sigma}\right)$,

(ii) $\left(\mathbf{G}_{\boldsymbol{\theta}_{1} \mid \mathscr{F}}+\mathbf{E} \mathbf{Q} \mathbf{W}\right) \mathbf{y}=\mathbf{G}_{\boldsymbol{\theta}_{1} \mid \mathscr{M}} \mathbf{y}$ for all $\mathbf{y} \in \mathscr{C}\left(\mathbf{W}_{m}\right)$ and for all $\mathbf{E}$,

(iii) $\mathbf{G}_{\boldsymbol{\theta}_{1} \mid \mathscr{F}}\left(\mathbf{X}_{1}: \mathbf{\Sigma} \mathbf{M}_{1}\right)=\left(\mathbf{M}_{2} \mathbf{X}_{1}: \mathbf{0}\right)$,

(iv) $\left\{\operatorname{BLUE}\left(\boldsymbol{\theta}_{1} \mid \mathscr{F}\right)\right\} \subseteq\left\{\operatorname{BLUE}\left(\boldsymbol{\theta}_{1} \mid \mathscr{M}\right)\right\}$.

\section{Equality of a particular BLUE and BLUP}

In this section we consider the relation

$$
\operatorname{BLUE}\left(\mathbf{M}_{2} \mathbf{X}_{1} \boldsymbol{\beta}_{1} \mid \mathscr{F}\right) \text { versus } \operatorname{BLUP}\left(\mathbf{M}_{2} \mathbf{X}_{1} \boldsymbol{\beta}_{1}+\mathbf{X}_{2} \mathbf{u} \mid \mathscr{M}\right) .
$$

Recall, by (3), that under $\mathscr{M}$ we have

$$
\begin{aligned}
\operatorname{BLUP}\left(\mathbf{M}_{2} \mathbf{X}_{1} \boldsymbol{\beta}+\mathbf{X}_{2} \mathbf{u}\right) & =\operatorname{BLUE}\left(\mathbf{M}_{2} \mathbf{X}_{1} \boldsymbol{\beta}_{1}\right)+\operatorname{BLUP}\left(\mathbf{X}_{2} \mathbf{u}\right) \\
& =\operatorname{BLUE}\left(\mathbf{M}_{2} \mathbf{X}_{1} \boldsymbol{\beta}_{1}\right)+\mathbf{X}_{2} \operatorname{BLUP}(\mathbf{u}) .
\end{aligned}
$$

By Lemma 1.1, Ly is the BLUP for $\boldsymbol{\eta}_{1}=\mathbf{M}_{2} \mathbf{X}_{1} \boldsymbol{\beta}_{1}+\mathbf{X}_{2} \mathbf{u}$ if and only if

$$
\mathbf{L}\left(\mathbf{X}_{1}: \mathbf{\Sigma} \mathbf{M}_{1}\right)=\left(\mathbf{M}_{2} \mathbf{X}_{1}: \mathbf{X}_{2} \mathbf{D} \mathbf{X}_{2}^{\prime} \mathbf{M}_{1}\right)
$$

where $\boldsymbol{\Sigma}=\mathbf{X}_{2} \mathbf{D} \mathbf{X}_{2}^{\prime}+\mathbf{V}$. The general solution to $\mathbf{L}$ in $(15)$ can be expressed as

$$
\begin{aligned}
\mathbf{L}_{0} & =\mathbf{M}_{2} \mathbf{X}_{1}\left(\mathbf{X}_{1}^{\prime} \mathbf{W}_{m}^{-} \mathbf{X}_{1}\right)^{-} \mathbf{X}_{1}^{\prime} \mathbf{W}_{m}^{+}+\mathbf{X}_{2} \mathbf{D} \mathbf{X}_{2}^{\prime} \mathbf{M}_{1}\left(\mathbf{M}_{1} \boldsymbol{\Sigma} \mathbf{M}_{1}\right)^{+} \mathbf{M}_{1}+\mathbf{E} \mathbf{Q}_{\mathbf{W}_{m}} \\
& =\mathbf{G}_{\boldsymbol{\theta}_{1} \mid \mathscr{M}}+\mathbf{G}_{\mathbf{X}_{2} \mathbf{u} \mid \mathscr{M}}+\mathbf{E} \mathbf{Q}_{\mathbf{W}_{m}},
\end{aligned}
$$

where $\mathbf{E} \in \mathbb{R}^{n \times n}$ is free to vary and $\mathbf{W}_{m}=\mathbf{X}_{1} \mathbf{X}_{1}^{\prime}+\boldsymbol{\Sigma}$. Suppose that $\mathbf{L}_{0}$ provides also the BLUE for $\boldsymbol{\theta}_{1}=\mathbf{M}_{2} \mathbf{X}_{1} \boldsymbol{\beta}_{1}$ under the fixed model $\mathscr{F}$. Then $\mathbf{L}_{0}$ has to satisfy, for every $\mathbf{E}$, the fundamental BLUE equation

$$
\mathbf{L}_{0}\left(\mathbf{X}_{1}: \mathbf{X}_{2}: \mathbf{V M}\right)=\mathbf{L}_{0}\left(\mathbf{X}_{1}: \mathbf{X}_{2}: \mathbf{\Sigma M}\right)=\left(\mathbf{M}_{2} \mathbf{X}_{1}: \mathbf{0}: \mathbf{0}\right) \text {. }
$$

Trivially the $\mathbf{X}_{1}$-part of 16 holds. Moreover, we must have

$$
\left(\mathbf{G}_{\boldsymbol{\theta}_{1} \mid \mathscr{M}}+\mathbf{G}_{\mathbf{X}_{2} \mathbf{u} \mid \mathscr{M}}+\mathbf{E Q}_{\mathbf{W}_{m}}\right)\left(\mathbf{X}_{2}: \mathbf{\Sigma} \mathbf{M}\right)=(\mathbf{0}: \mathbf{0}) \text { for all } \mathbf{E},
$$


which implies that $\mathscr{C}\left(\mathbf{X}_{2}\right) \subseteq \mathscr{C}\left(\mathbf{W}_{m}\right)=\mathscr{C}\left(\mathbf{X}_{1}: \mathbf{\Sigma} \mathbf{M}_{1}\right)$, and thereby

$$
\mathscr{C}(\mathbf{W})=\mathscr{C}\left(\mathbf{W}_{m}\right), \quad \mathbf{X}_{2}=\mathbf{X}_{1} \mathbf{A}+\mathbf{\Sigma} \mathbf{M}_{1} \mathbf{B}=\mathbf{X}_{1} \mathbf{A}+\mathbf{W}_{m} \mathbf{M}_{1} \mathbf{B}
$$

for some $\mathbf{A}$ and $\mathbf{B}$. We further must have

$$
\left(\mathbf{G}_{\boldsymbol{\theta}_{1} \mid \mathscr{M}}+\mathbf{G}_{\mathbf{X}_{2} \mathbf{u} \mid \mathscr{M}}\right)\left(\mathbf{X}_{2}: \mathbf{\Sigma} \mathbf{M}\right)=(\mathbf{0}: \mathbf{0}) .
$$

Consider first the $\boldsymbol{\Sigma} \mathbf{M}$-part of (18). In view of 15 we have

$$
\left(\mathbf{G}_{\boldsymbol{\theta}_{1} \mid \mathscr{M}}+\mathbf{G}_{\mathbf{X}_{2} \mathbf{u} \mid \mathscr{M}}\right) \boldsymbol{\Sigma} \mathbf{M}_{1}=\mathbf{X}_{2} \mathbf{D} \mathbf{X}_{2}^{\prime} \mathbf{M}_{1}
$$

which further implies

$$
\left(\mathbf{G}_{\boldsymbol{\theta}_{1} \mid \mathscr{M}}+\mathbf{G}_{\mathbf{X}_{2} \mathbf{u} \mid \mathscr{M}}\right) \boldsymbol{\Sigma} \mathbf{M}_{1} \mathbf{Q}_{\mathbf{M}_{1} \mathbf{X}_{2}}=\mathbf{X}_{2} \mathbf{D} \mathbf{X}_{2}^{\prime} \mathbf{M}_{1} \mathbf{Q}_{\mathbf{M}_{1} \mathbf{X}_{2}}=\mathbf{0}
$$

i.e., $\left(\mathbf{G}_{\boldsymbol{\theta}_{1} \mid \mathscr{M}}+\mathbf{G}_{\mathbf{X}_{2} \mathbf{u} \mid \mathscr{M}}\right) \mathbf{\Sigma} \mathbf{M}=\mathbf{0}$, and thereby $\boldsymbol{\Sigma} \mathbf{M}$-part of (18) holds.

For the $\mathbf{X}_{2}$-part in 18 we must have

$$
\begin{aligned}
\left(\mathbf{G}_{\boldsymbol{\theta}_{1} \mid \mathscr{M}}+\mathbf{G}_{\mathbf{X}_{2} \mathbf{u} \mid \mathscr{M}}\right) \mathbf{X}_{2}= & \mathbf{M}_{2} \mathbf{X}_{1}\left(\mathbf{X}_{1}^{\prime} \mathbf{W}_{m}^{-} \mathbf{X}_{1}\right)^{-} \mathbf{X}_{1}^{\prime} \mathbf{W}_{m}^{+} \mathbf{X}_{2} \\
& +\mathbf{X}_{2} \mathbf{D} \mathbf{X}_{2}^{\prime} \mathbf{M}_{1}\left(\mathbf{M}_{1} \boldsymbol{\Sigma} \mathbf{M}_{1}\right)^{+} \mathbf{M}_{1} \mathbf{X}_{2}=\mathbf{0}
\end{aligned}
$$

which clearly holds if and only if

$$
\begin{aligned}
\mathbf{G}_{\boldsymbol{\theta}_{1} \mid \mathscr{M}} \mathbf{X}_{2} & =\mathbf{M}_{2} \mathbf{X}_{1}\left(\mathbf{X}_{1}^{\prime} \mathbf{W}_{m}^{-} \mathbf{X}_{1}\right)^{-} \mathbf{X}_{1}^{\prime} \mathbf{W}_{m}^{+} \mathbf{X}_{2}=\mathbf{0} \\
\mathbf{G}_{\mathbf{X}_{2} \mathbf{u} \mid \mathscr{M}} \mathbf{X}_{2} & =\mathbf{X}_{2} \mathbf{D} \mathbf{X}_{2}^{\prime} \mathbf{M}_{1}\left(\mathbf{M}_{1} \boldsymbol{\Sigma} \mathbf{M}_{1}\right)^{+} \mathbf{M}_{1} \mathbf{X}_{2}=\mathbf{0}
\end{aligned}
$$

Substituting $\mathbf{X}_{2}=\mathbf{X}_{1} \mathbf{A}+\mathbf{W}_{m} \mathbf{M}_{1} \mathbf{B}$ into 20 a yields $\mathbf{M}_{2} \mathbf{X}_{1} \mathbf{A}=\mathbf{0}$, so that $\mathbf{A}=\mathbf{Q}_{\mathbf{X}_{1}^{\prime} \mathbf{M}_{2}} \mathbf{Z}$ for some $\mathbf{Z}$, and thereby, taking (17) into account,

$$
\mathbf{X}_{2}=\mathbf{X}_{1} \mathbf{Q}_{\mathbf{X}_{1}^{\prime} \mathbf{M}_{2}} \mathbf{Z}+\mathbf{\Sigma} \mathbf{M}_{1} \mathbf{B}
$$

Moreover, by part (f) of Lemma 1.2 , we have

$$
\mathscr{C}\left(\mathbf{X}_{1} \mathbf{Q}_{\mathbf{X}_{1}^{\prime} \mathbf{M}_{2}}\right)=\mathscr{C}\left[\mathbf{X}_{1}\left(\mathbf{X}_{1}^{\prime} \mathbf{X}_{2}^{\perp}\right)^{\perp}\right]=\mathscr{C}\left(\mathbf{X}_{1}\right) \cap \mathscr{C}\left(\mathbf{X}_{2}\right) .
$$

Consider then (20b). Substituting (21) into (20b) yields

$$
\mathbf{X}_{2} \mathbf{D X}_{2}^{\prime} \mathbf{M}_{1}\left(\mathbf{M}_{1} \Sigma \mathbf{M}_{1}\right)^{+} \mathbf{M}_{1} \Sigma \mathbf{M}_{1} \mathbf{B}=\mathbf{0}
$$

i.e., $\mathbf{X}_{2} \mathbf{D} \mathbf{X}_{2}^{\prime} \mathbf{M}_{1} \mathbf{B}=\mathbf{0}$, so that $\mathscr{C}(\mathbf{B}) \subseteq \mathscr{C}\left(\mathbf{M}_{1} \mathbf{X}_{2} \mathbf{D} \mathbf{X}_{2}^{\prime}\right)^{\perp}$, and by 21,

$$
\mathscr{C}\left(\mathbf{X}_{2}\right) \subseteq \mathscr{C}\left(\mathbf{X}_{1} \mathbf{Q}_{\mathbf{X}_{1}^{\prime} \mathbf{M}_{2}}: \mathbf{\Sigma} \mathbf{M}_{1} \mathbf{Q}_{\mathbf{M}_{1} \mathbf{X}_{2} \mathbf{D} \mathbf{X}_{2}^{\prime}}\right) .
$$

In light of part (f) of Lemma 1.2 we can further write

$$
\mathscr{C}\left(\mathbf{M}_{1} \mathbf{Q}_{\mathbf{M}_{1} \mathbf{X}_{2} \mathbf{D} \mathbf{X}_{2}^{\prime}}\right)=\mathscr{C}\left(\mathbf{X}_{1}: \mathbf{X}_{2} \mathbf{D} \mathbf{X}_{2}^{\prime}\right)^{\perp}=\mathscr{C}\left(\mathbf{X}_{1}: \mathbf{M}_{1} \mathbf{X}_{2} \mathbf{D} \mathbf{X}_{2}^{\prime}\right)^{\perp}
$$

Thus, noting that $\mathbf{M}_{1} \mathbf{X}_{2} \mathbf{D} \mathbf{X}_{2}^{\prime}=\mathbf{M}_{1}(\boldsymbol{\Sigma}-\mathbf{V})$, we have obtained the following theorem. 
Theorem 3.1. An arbitrary BLUP for $\boldsymbol{\eta}_{1}=\mathbf{M}_{2} \mathbf{X}_{1} \boldsymbol{\beta}+\mathbf{X}_{2} \mathbf{u}$ under $\mathscr{M}$ provides also the BLUE for $\boldsymbol{\theta}_{1}=\mathbf{M}_{2} \mathbf{X}_{1} \boldsymbol{\beta}_{1}$ under the fixed model $\mathscr{F}$, i.e.,

$\left\{\operatorname{BLUP}\left(\mathbf{M}_{2} \mathbf{X}_{1} \boldsymbol{\beta}_{1}+\mathbf{X}_{2} \mathbf{u} \mid \mathscr{M}\right)\right\} \subseteq\left\{\operatorname{BLUE}\left(\mathbf{M}_{2} \mathbf{X}_{1} \boldsymbol{\beta}_{1} \mid \mathscr{F}\right)\right\}$,

i.e., $\left\{\mathbf{P}_{\boldsymbol{\eta}_{1} \mid \mathscr{M}} \subseteq\left\{\mathbf{P}_{\boldsymbol{\theta}_{1} \mid \mathscr{F}}\right\}\right.$, if and only if

$$
\mathscr{C}\left(\mathbf{X}_{2}\right) \subseteq \mathscr{C}\left(\mathbf{R}: \mathbf{\Sigma} \mathbf{M}_{1} \mathbf{S}\right)
$$

where the matrices $\mathbf{R}$ and $\mathbf{S}$ have properties $\mathscr{C}(\mathbf{R})=\mathscr{C}\left(\mathbf{X}_{1}\right) \cap \mathscr{C}\left(\mathbf{X}_{2}\right)$ and

$$
\mathscr{C}(\mathbf{S})=\mathscr{C}\left[\mathbf{X}_{1}: \mathbf{M}_{1}(\mathbf{\Sigma}-\mathbf{V})\right]^{\perp}=\mathscr{C}\left(\mathbf{X}_{1}: \mathbf{M}_{1} \mathbf{X}_{2} \mathbf{D} \mathbf{X}_{2}^{\prime}\right)^{\perp}
$$

The reverse inclusion to 22 is considered in Theorem 3.2 .

Theorem 3.2. An arbitrary BLUE for $\boldsymbol{\theta}_{1}=\mathbf{M}_{2} \mathbf{X}_{1} \boldsymbol{\beta}_{1}$ under $\mathscr{F}$ provides also the BLUP for $\boldsymbol{\eta}_{1}=\mathbf{M}_{2} \mathbf{X}_{1} \boldsymbol{\beta}_{1}+\mathbf{X}_{2} \mathbf{u}$ under the mixed model $\mathscr{M}$, i.e.,

$$
\left\{\operatorname{BLUE}\left(\mathbf{M}_{2} \mathbf{X}_{1} \boldsymbol{\beta}_{1} \mid \mathscr{F}\right)\right\} \subseteq\left\{\operatorname{BLUP}\left(\mathbf{M}_{2} \mathbf{X}_{1} \boldsymbol{\beta}_{1}+\mathbf{X}_{2} \mathbf{u} \mid \mathscr{M}\right)\right\},
$$

i.e., $\left\{\mathbf{P}_{\boldsymbol{\theta}_{1} \mid \mathscr{F}}\right\} \subseteq\left\{\mathbf{P}_{\boldsymbol{\eta}_{1}, \mid \mathscr{M}}\right\}$, if and only if the following two conditions hold:

(a) $\mathscr{C}\left(\boldsymbol{\Sigma M}_{1}\right) \subseteq \mathscr{C}\left(\mathbf{X}_{2}: \boldsymbol{\Sigma} \mathbf{M}\right)$, i.e., $\left\{\operatorname{BLUE}\left(\boldsymbol{\theta}_{1} \mid \mathscr{F}\right)\right\} \subseteq\left\{\operatorname{BLUE}\left(\boldsymbol{\theta}_{1} \mid \mathscr{M}\right)\right\}$,

(b) $\mathbf{\Sigma} \mathbf{M}_{1}=\mathbf{V M}_{1}$, i.e., $\mathbf{M}_{1} \mathbf{X}_{2} \mathbf{D} \mathbf{X}_{2}^{\prime}=\mathbf{0}$.

Proof. Take an arbitrary member in the class $\left\{\mathbf{P}_{\boldsymbol{\theta}_{1} \mid \mathscr{F}}\right\}$,

$$
\mathbf{B}_{0}=\mathbf{G}_{\boldsymbol{\theta}_{1} \mid \mathscr{F}}+\mathbf{E} \mathbf{Q}_{\mathbf{W}}=\mathbf{M}_{2} \mathbf{X}_{1}\left(\mathbf{X}_{1}^{\prime} \dot{\mathbf{M}}_{2} \mathbf{X}_{1}\right)^{-} \mathbf{X}_{1}^{\prime} \dot{\mathbf{M}}_{2}+\mathbf{E} \mathbf{Q}_{\mathbf{W}}
$$

and $\mathbf{E}$ is free to vary and $\mathscr{C}(\mathbf{W})=\mathscr{C}\left(\mathbf{X}_{1}: \mathbf{X}_{2}: \mathbf{V}\right)$. Then $\mathbf{B}_{0}$ provides the BLUP for $\boldsymbol{\eta}_{1}=\mathbf{M}_{2} \mathbf{X}_{1} \boldsymbol{\beta}_{1}+\mathbf{X}_{2} \mathbf{u}$ under the mixed model $\mathscr{M}$ if and only if

$$
\left(\mathbf{G}_{\boldsymbol{\theta}_{1} \mid \mathscr{F}}+\mathbf{E} \mathbf{Q}_{\mathbf{W}}\right)\left(\mathbf{X}_{1}: \mathbf{\Sigma} \mathbf{M}_{1}\right)=\left(\mathbf{M}_{2} \mathbf{X}_{1}: \mathbf{X}_{2} \mathbf{D} \mathbf{X}_{2}^{\prime} \mathbf{M}_{1}\right)
$$

holds for every $\mathbf{E}$. The $\mathbf{X}_{1}$-part is clear. The $\mathbf{\Sigma} \mathbf{M}_{1}$-part is

$$
\left(\mathbf{G}_{\boldsymbol{\theta}_{1} \mid \mathscr{F}}+\mathbf{E} \mathbf{Q}_{\mathbf{W}}\right) \mathbf{\Sigma} \mathbf{M}_{1}=\mathbf{X}_{2} \mathbf{D} \mathbf{X}_{2}^{\prime} \mathbf{M}_{1}
$$

i.e.,

$$
\mathbf{G}_{\boldsymbol{\theta}_{1} \mid \mathscr{F}} \boldsymbol{\Sigma} \mathbf{M}_{1}=\mathbf{M}_{2} \mathbf{X}_{1}\left(\mathbf{X}_{1}^{\prime} \dot{\mathbf{M}}_{2} \mathbf{X}_{1}\right)^{-} \mathbf{X}_{1}^{\prime} \dot{\mathbf{M}}_{2} \boldsymbol{\Sigma} \mathbf{M}_{1}=\mathbf{X}_{2} \mathbf{D} \mathbf{X}_{2}^{\prime} \mathbf{M}_{1}
$$

It is clear that 25) holds if and only if

$$
\begin{aligned}
\mathbf{M}_{2} \mathbf{X}_{1}\left(\mathbf{X}_{1}^{\prime} \dot{\mathbf{M}}_{2} \mathbf{X}_{1}\right)^{-} \mathbf{X}_{1}^{\prime} \dot{\mathbf{M}}_{2} \boldsymbol{\Sigma} \mathbf{M}_{1}=\mathbf{0} \\
\mathbf{X}_{2} \mathbf{D} \mathbf{X}_{2}^{\prime} \mathbf{M}_{1}=\mathbf{0}
\end{aligned}
$$

where $26 \mathrm{a}$ is equivalent to

$$
\mathbf{X}_{1}^{\prime} \dot{\mathbf{M}}_{2} \boldsymbol{\Sigma} \mathbf{M}_{1}=\mathbf{0}, \quad \text { i.e., } \quad \mathscr{C}\left(\boldsymbol{\Sigma} \mathbf{M}_{1}\right) \subseteq \mathscr{C}\left(\dot{\mathbf{M}}_{2} \mathbf{X}_{1}\right)^{\perp} .
$$

In view of $\mathscr{C}\left(\mathbf{\Sigma} \mathbf{M}_{1}\right) \subseteq \mathscr{C}(\mathbf{W})$, 27) can be written equivalently as

$$
\mathscr{C}\left(\mathbf{\Sigma} \mathbf{M}_{1}\right) \subseteq \mathscr{C}\left(\dot{\mathbf{M}}_{2} \mathbf{X}_{1}\right)^{\perp} \cap \mathscr{C}(\mathbf{W}) .
$$

On the other hand, in light of part (e) of Lemma 1.2 we know that

$$
\mathscr{C}\left(\dot{\mathbf{M}}_{2} \mathbf{X}_{1}: \mathbf{Q} \mathbf{W}\right)^{\perp}=\mathscr{C}\left(\dot{\mathbf{M}}_{2} \mathbf{X}_{1}\right)^{\perp} \cap \mathscr{C}(\mathbf{W})=\mathscr{C}\left(\mathbf{X}_{2}: \mathbf{V M}\right) .
$$


Combining (28) and (29) gives

$$
\mathscr{C}\left(\boldsymbol{\Sigma} \mathbf{M}_{1}\right) \subseteq \mathscr{C}\left(\mathbf{X}_{2}: \mathbf{V M}\right)=\mathscr{C}\left(\mathbf{X}_{2}: \mathbf{\Sigma} \mathbf{M}\right)
$$

Moreover, 26b is equivalent to $\mathbf{V M}_{1}=\mathbf{\Sigma M}_{1}$, which completes the proof.

What about the equality of the sets $\left\{\mathbf{P}_{\boldsymbol{\theta}_{1} \mid \mathscr{F}}\right\}$ and $\left\{\mathbf{P}_{\boldsymbol{\eta}_{1} \mid \mathscr{M}}\right\}$ ? Requesting that (b) of Theorem 3.2 holds, i.e., $\mathbf{V M}_{1}=\boldsymbol{\Sigma} \mathbf{M}_{1}$, the condition (23) of Theorem 3.1 becomes $\mathscr{C}\left(\mathbf{X}_{2}\right) \subset \mathscr{C}\left(\mathbf{R}: \boldsymbol{\Sigma} \mathbf{M}_{1}\right)$, i.e.,

$$
\mathscr{C}\left(\mathbf{X}_{2}: \mathbf{\Sigma} \mathbf{M}\right) \subseteq \mathscr{C}\left(\mathbf{R}: \mathbf{\Sigma} \mathbf{M}_{1}\right) \text {, where } \mathscr{C}(\mathbf{R})=\mathscr{C}\left(\mathbf{X}_{1}\right) \cap \mathscr{C}\left(\mathbf{X}_{2}\right) .
$$

On the other hand, condition (a) of Theorem 3.2 is equivalent to

$$
\mathscr{C}\left(\mathbf{R}: \mathbf{\Sigma} \mathbf{M}_{1}\right) \subseteq \mathscr{C}\left(\mathbf{X}_{2}: \mathbf{\Sigma M}\right) .
$$

Now (30) and (31) imply the following result.

Corollary 3.1. The following statements are equivalent:

(a) $\left\{\operatorname{BLUP}\left(\boldsymbol{\theta}_{1}+\mathbf{X}_{2} \mathbf{u} \mid \mathscr{M}\right)\right\}=\left\{\operatorname{BLUE}\left(\boldsymbol{\theta}_{1} \mid \mathscr{F}\right)\right\}$,

(b) $\mathscr{C}\left(\mathbf{X}_{2}: \mathbf{\Sigma} \mathbf{M}\right)=\mathscr{C}\left(\mathbf{R}: \mathbf{\Sigma} \mathbf{M}_{1}\right)$ and $\mathbf{M}_{1} \mathbf{X}_{2} \mathbf{D} \mathbf{X}_{2}^{\prime}=\mathbf{0}$, i.e., $\mathbf{\Sigma} \mathbf{M}_{1}=$ $\mathbf{V M}_{1}$, where $\mathscr{C}(\mathbf{R})=\mathscr{C}\left(\mathbf{X}_{1}\right) \cap \mathscr{C}\left(\mathbf{X}_{2}\right)$.

Notice that if $\boldsymbol{\mu}_{1}$ is estimable in $\mathscr{F}$ then $\mathbf{M}_{1} \mathbf{X}_{2} \mathbf{D} \mathbf{X}_{2}^{\prime}=\mathbf{0}$ is equivalent to $\mathbf{X}_{2} \mathbf{D} \mathbf{X}_{2}^{\prime}=\mathbf{0}$. Moreover, from Corollary 3.1 we can conclude the following.

Corollary 3.2. Suppose that $\boldsymbol{\mu}_{1}=\mathbf{X}_{1} \boldsymbol{\beta}_{1}$ is estimable under $\mathscr{F}$. Then the following three statements are equivalent:

(a) $\left\{\operatorname{BLUP}\left(\boldsymbol{\mu}_{1}+\mathbf{X}_{2} \mathbf{u} \mid \mathscr{M}\right)\right\}=\left\{\operatorname{BLUE}\left(\boldsymbol{\mu}_{1} \mid \mathscr{F}\right)\right\}$,

(b) $\left\{\operatorname{BLUE}\left(\boldsymbol{\mu}_{1} \mid \mathscr{M}\right)\right\}=\left\{\operatorname{BLUE}\left(\boldsymbol{\mu}_{1} \mid \mathscr{F}\right)\right\}$ and $\mathbf{X}_{2} \mathbf{D X}_{2}^{\prime}=\mathbf{0}$, i.e., $\boldsymbol{\Sigma}=\mathbf{V}$,

(c) $\mathscr{C}\left(\boldsymbol{\Sigma} \mathbf{M}_{1}\right)=\mathscr{C}\left(\mathbf{X}_{2}: \mathbf{V M}\right)$ and $\mathbf{X}_{2} \mathbf{D} \mathbf{X}_{2}^{\prime}=\mathbf{0}$.

Remark 3.1. The property $\operatorname{cov}\left(\mathbf{X}_{2} \mathbf{u}\right)=\mathbf{X}_{2} \mathbf{D} \mathbf{X}_{2}^{\prime}=\mathbf{0}$ together with $\mathrm{E}(\mathbf{u})=$ $\mathbf{0}$ means that $\mathbf{X}_{2} \mathbf{u}=\mathbf{0}$ with probability 1 . Moreover, if $\mathbf{X}_{2} \mathbf{D} \mathbf{X}_{2}^{\prime}=\mathbf{0}$, then the mixed model $\mathscr{M}$ becomes the small fixed model $\mathscr{F}_{1}=\left\{\mathbf{y}, \mathbf{X}_{1} \boldsymbol{\beta}_{1}, \mathbf{V}\right\}$ and then any of the conditions in Corollary 3.2 implies the equality

$$
\left\{\operatorname{BLUE}\left(\boldsymbol{\mu}_{1} \mid \mathscr{F}_{1}\right)\right\}=\left\{\operatorname{BLUE}\left(\boldsymbol{\mu}_{1} \mid \mathscr{F}\right)\right\},
$$

which further is equivalent to $\mathscr{C}\left(\mathbf{V M}_{1}\right)=\mathscr{C}\left(\mathbf{X}_{2}: \mathbf{V M}\right)$.

\section{A further equality of particular BLUE and BLUP}

In this section we consider

$$
\operatorname{BLUE}\left(\mathbf{M}_{1} \mathbf{X}_{2} \boldsymbol{\beta}_{2} \mid \mathscr{F}\right) \text { versus } \operatorname{BLUP}\left(\mathbf{M}_{1} \mathbf{X}_{2} \mathbf{u} \mid \mathscr{M}\right) \text {. }
$$

Theorem 4.1. An arbitrary BLUP for $\mathbf{M}_{1} \mathbf{X}_{2} \mathbf{u}$ under $\mathscr{M}$ provides also the BLUE for $\boldsymbol{\theta}_{2}=\mathbf{M}_{1} \mathbf{X}_{2} \boldsymbol{\beta}_{2}$ under the fixed model $\mathscr{F}$, i.e.,

$$
\left\{\operatorname{BLUP}\left(\mathbf{M}_{1} \mathbf{X}_{2} \mathbf{u} \mid \mathscr{M}\right)\right\} \subseteq\left\{\operatorname{BLUE}\left(\mathbf{M}_{1} \mathbf{X}_{2} \boldsymbol{\beta}_{2} \mid \mathscr{F}\right)\right\},
$$




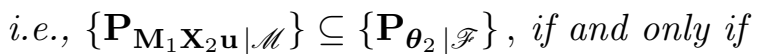

$$
\mathscr{C}\left(\mathbf{X}_{2}\right) \subseteq \mathscr{C}\left(\mathbf{X}_{1}: \mathbf{\Sigma} \mathbf{M}_{1} \mathbf{Q}_{\mathbf{M}_{1} \mathbf{V}}\right)=\mathscr{C}\left(\mathbf{X}_{1}: \boldsymbol{\Sigma} \mathbf{M}_{1} \mathbf{Q}_{\left(\mathbf{X}_{1}: \mathbf{V}\right)}\right) \text {. }
$$

Proof. We recall that $\mathbf{C y}$ is the BLUP for $\mathbf{M}_{1} \mathbf{X}_{2} \mathbf{u}$ under $\mathscr{M}$ if and only if

$$
\mathbf{C}\left(\mathbf{X}_{1}: \mathbf{\Sigma} \mathbf{M}_{1}\right)=\left(\mathbf{0}: \mathbf{M}_{1} \mathbf{X}_{2} \mathbf{D X} \mathbf{X}_{2}^{\prime} \mathbf{M}_{1}\right) .
$$

The general solution to $\mathbf{C}$ in $(33)$ is

$$
\begin{aligned}
\mathbf{C}_{0} & =\mathbf{M}_{1} \mathbf{X}_{2} \mathbf{D} \mathbf{X}_{2}^{\prime} \mathbf{M}_{1}\left(\mathbf{M}_{1} \boldsymbol{\Sigma} \mathbf{M}_{1}\right)^{+} \mathbf{M}_{1}+\mathbf{E Q}_{\mathbf{W}_{m}} \\
& =\mathbf{G}_{\mathbf{M}_{1} \mathbf{X}_{2} \mathbf{u} \mid \mathscr{M}}+\mathbf{E} \mathbf{Q}_{\mathbf{W}_{m}},
\end{aligned}
$$

where $\mathbf{E}$ is free to vary and $\mathbf{W}_{m}=\mathbf{X}_{1} \mathbf{X}_{1}^{\prime}+\boldsymbol{\Sigma}$. Suppose that $\mathbf{C}_{0}$ provides also the BLUE for $\boldsymbol{\theta}_{2}=\mathbf{M}_{1} \mathbf{X}_{2} \boldsymbol{\beta}_{2}$ under the fixed model $\mathscr{F}$. Then $\mathbf{C}_{0}$ has to satisfy, for every $\mathbf{E}$, the fundamental BLUE equation

$$
\left(\mathbf{G}_{\mathbf{M}_{1} \mathbf{X}_{2} \mathbf{u} \mid \mathscr{M}}+\mathbf{E Q}_{\mathbf{W}_{m}}\right)\left(\mathbf{X}_{1}: \mathbf{X}_{2}: \mathbf{V M}\right)=\left(\mathbf{0}: \mathbf{M}_{1} \mathbf{X}_{2}: \mathbf{0}\right) \text {. }
$$

By (33) the $\mathbf{X}_{1}$-part of (34) holds. Moreover, we must have

$$
\left(\mathbf{G}_{\mathbf{M}_{1} \mathbf{X}_{2} \mathbf{u} \mid \mathscr{M}}+\mathbf{E} \mathbf{Q}_{\mathbf{W}_{m}}\right)\left(\mathbf{X}_{2}: \mathbf{V M}\right)=\left(\mathbf{M}_{1} \mathbf{X}_{2}: \mathbf{0}\right) \text { for all } \mathbf{E},
$$

from which it follows that $\mathscr{C}\left(\mathbf{X}_{2}\right) \subseteq \mathscr{C}\left(\mathbf{W}_{m}\right)=\mathscr{C}\left(\mathbf{X}_{1}: \mathbf{\Sigma}\right)$, and hence

$$
\mathscr{C}(\mathbf{W})=\mathscr{C}\left(\mathbf{W}_{m}\right) \text { and } \mathbf{X}_{2}=\mathbf{X}_{1} \mathbf{A}+\boldsymbol{\Sigma} \mathbf{M}_{1} \mathbf{B}
$$

for some $\mathbf{A}$ and $\mathbf{B}$. We further must have

$$
\mathbf{G}_{\mathbf{M}_{1} \mathbf{X}_{2} \mathbf{u} \mid \mathscr{M}}\left(\mathbf{X}_{2}: \mathbf{V M}\right)=\left(\mathbf{M}_{1} \mathbf{X}_{2}: \mathbf{0}\right) .
$$

Using $\mathbf{V M}=\boldsymbol{\Sigma} \mathbf{M}$, (36) can be written as

$$
\begin{aligned}
\mathbf{G}_{\mathbf{M}_{1} \mathbf{X}_{2} \mathbf{u} \mid \mathscr{M}} \mathbf{X}_{2} & =\mathbf{M}_{1} \mathbf{X}_{2} \mathbf{D} \mathbf{X}_{2}^{\prime} \mathbf{M}_{1}\left(\mathbf{M}_{1} \Sigma \mathbf{M}_{1}\right)^{+} \mathbf{M}_{1} \mathbf{X}_{2}=\mathbf{M}_{1} \mathbf{X}_{2}, \\
\mathbf{G}_{\mathbf{M}_{1} \mathbf{X}_{2} \mathbf{u} \mid \mathscr{M}} \mathbf{\Sigma} \mathbf{M} & =\mathbf{M}_{1} \mathbf{X}_{2} \mathbf{D} \mathbf{X}_{2}^{\prime} \mathbf{M}_{1}\left(\mathbf{M}_{1} \Sigma \mathbf{M}_{1}\right)^{+} \mathbf{M}_{1} \boldsymbol{\Sigma} \mathbf{M}=\mathbf{0} .
\end{aligned}
$$

Now (37b) can be expressed as

$$
\mathbf{M}_{1} \mathbf{X}_{2} \mathbf{D X} \mathbf{X}_{2}^{\prime} \mathbf{M}_{1}\left(\mathbf{M}_{1} \boldsymbol{\Sigma} \mathbf{M}_{1}\right)^{+} \mathbf{M}_{1} \boldsymbol{\Sigma} \mathbf{M}_{1} \mathbf{Q}_{\mathbf{M}_{1} \mathbf{X}_{2}}=\mathbf{0},
$$

which obviously holds.

Consider then (37a):

$$
\mathbf{M}_{1}(\boldsymbol{\Sigma}-\mathbf{V}) \mathbf{M}_{1}\left(\mathbf{M}_{1} \boldsymbol{\Sigma} \mathbf{M}_{1}\right)^{+} \mathbf{M}_{1} \mathbf{X}_{2}=\mathbf{M}_{1} \mathbf{X}_{2}
$$

from which, in view of $\mathscr{C}\left(\mathbf{M}_{1} \mathbf{X}_{2}\right) \subseteq \mathscr{C}\left(\mathbf{M}_{1} \boldsymbol{\Sigma}\right)$, it follows that

$$
\mathbf{M}_{1} \mathbf{X}_{2}-\mathbf{M}_{1} \mathbf{V M}_{1}\left(\mathbf{M}_{1} \boldsymbol{\Sigma} \mathbf{M}_{1}\right)^{+} \mathbf{M}_{1} \mathbf{X}_{2}=\mathbf{M}_{1} \mathbf{X}_{2}
$$

i.e.,

$$
\mathbf{V M}_{1}\left(\mathbf{M}_{1} \boldsymbol{\Sigma} \mathbf{M}_{1}\right)^{+} \mathbf{M}_{1} \mathbf{X}_{2}=\mathbf{0} .
$$

Substituting $\mathbf{X}_{2}=\mathbf{X}_{1} \mathbf{A}+\boldsymbol{\Sigma} \mathbf{M}_{1} \mathbf{B}$ into (35) yields $\mathbf{V M}_{1} \mathbf{B}=\mathbf{0}$, so that $\mathscr{C}(\mathbf{B}) \subseteq \mathscr{C}\left(\mathbf{M}_{1} \mathbf{V}\right)^{\perp}$ and thereby

$$
\mathscr{C}\left(\mathbf{X}_{2}\right) \subseteq \mathscr{C}\left(\mathbf{X}_{1}: \mathbf{\Sigma} \mathbf{M}_{1} \mathbf{Q}_{\mathbf{M}_{1} \mathbf{V}}\right)=\mathscr{C}\left(\mathbf{X}_{1}: \mathbf{\Sigma} \mathbf{M}_{1} \mathbf{Q}_{\left(\mathbf{X}_{1}: \mathbf{V}\right)}\right),
$$


where by part (f) of Lemma $1.2, \mathscr{C}\left(\mathbf{M}_{1} \mathbf{Q}_{\mathbf{M}_{1} \mathbf{V}}\right)=\mathscr{C}\left(\mathbf{X}_{1}: \mathbf{V}\right)^{\perp}$.

Let us consider the reverse inclusion to 32 ).

Theorem 4.2. An arbitrary BLUE for $\boldsymbol{\theta}_{2}=\mathbf{M}_{1} \mathbf{X}_{2} \boldsymbol{\beta}_{2}$ under $\mathscr{F}$ provides also the BLUP for $\mathbf{M}_{1} \mathbf{X}_{2} \mathbf{u}$ under the mixed model $\mathscr{M}$, i.e.,

$$
\begin{array}{r}
\left\{\operatorname{BLUE}\left(\mathbf{M}_{1} \mathbf{X}_{2} \boldsymbol{\beta}_{2} \mid \mathscr{F}\right)\right\} \subseteq\left\{\operatorname{BLUP}\left(\mathbf{M}_{1} \mathbf{X}_{2} \mathbf{u} \mid \mathscr{M}\right)\right\}, \\
\text { i.e., }\left\{\mathbf{P}_{\boldsymbol{\theta}_{2} \mid \mathscr{F}}\right\} \subseteq\left\{\mathbf{P}_{\left.\mathbf{M}_{1} \mathbf{X}_{2} \mathbf{u} \mid \mathscr{M}\right\}}\right\} \text { if and only if } \\
\mathscr{C}\left(\mathbf{V M}_{1}\right)=\mathscr{C}(\mathbf{V M}) .
\end{array}
$$

Proof. Take an arbitrary member in the class $\left\{\mathbf{P}_{\boldsymbol{\theta}_{2} \mid \mathscr{F}}\right\}$,

$$
\mathbf{N}_{0}=\mathbf{G}_{\boldsymbol{\theta}_{2} \mid \mathscr{F}}+\mathbf{E} \mathbf{Q}_{\mathbf{W}}=\mathbf{M}_{1} \mathbf{X}_{2}\left(\mathbf{X}_{2}^{\prime} \dot{\mathbf{M}}_{1} \mathbf{X}_{2}\right)^{-} \mathbf{X}_{2}^{\prime} \dot{\mathbf{M}}_{1}+\mathbf{E} \mathbf{Q}_{\mathbf{W}},
$$

where $\mathbf{E}$ is free to vary and $\mathscr{C}(\mathbf{W})=\mathscr{C}\left(\mathbf{X}_{1}: \mathbf{X}_{2}: \mathbf{V}\right)$. Then $\mathbf{N}_{0}$ provides the BLUP for $\mathbf{M}_{1} \mathbf{X}_{2} \mathbf{u}$ under the mixed model $\mathscr{M}$ if and only if

$$
\left(\mathbf{G}_{\boldsymbol{\theta}_{2} \mid \mathscr{F}}+\mathbf{E} \mathbf{Q}_{\mathbf{W}}\right)\left(\mathbf{X}_{1}: \mathbf{\Sigma} \mathbf{M}_{1}\right)=\left(\mathbf{0}: \mathbf{M}_{1} \mathbf{X}_{2} \mathbf{D X}_{2}^{\prime} \mathbf{M}_{1}\right)
$$

where the $\mathbf{X}_{1}$-part obviously holds and so we must have

$$
\mathbf{G}_{\boldsymbol{\theta}_{2} \mid \mathscr{F}} \boldsymbol{\Sigma} \mathbf{M}_{1}=\mathbf{M}_{1} \mathbf{X}_{2}\left(\mathbf{X}_{2}^{\prime} \dot{\mathbf{M}}_{1} \mathbf{X}_{2}\right)^{-} \mathbf{X}_{2}^{\prime} \dot{\mathbf{M}}_{1} \boldsymbol{\Sigma} \mathbf{M}_{1}=\mathbf{M}_{1} \mathbf{X}_{2} \mathbf{D} \mathbf{X}_{2}^{\prime} \mathbf{M}_{1} \text {. }
$$

Premultiplying (40) by $\mathbf{X}_{2}^{\prime} \dot{\mathbf{M}}_{1}$ yields an equivalent equation

$$
\mathbf{X}_{2}^{\prime} \dot{\mathbf{M}}_{1} \Sigma \mathbf{M}_{1}=\mathbf{X}_{2}^{\prime} \dot{\mathbf{M}}_{1} \mathbf{X}_{2} \mathbf{D} \mathbf{X}_{2}^{\prime} \mathbf{M}_{1}=\mathbf{X}_{2}^{\prime} \dot{\mathbf{M}}_{1}(\boldsymbol{\Sigma}-\mathbf{V}) \mathbf{M}_{1}
$$

i.e., $\mathbf{X}_{2}^{\prime} \dot{\mathbf{M}}_{1} \mathbf{V M}_{1}=\mathbf{0}$, which means that

$$
\mathscr{C}\left(\mathbf{V M}_{1}\right) \subseteq \mathscr{C}\left(\dot{\mathbf{M}}_{1} \mathbf{X}_{2}\right)^{\perp}
$$

We know that $\mathscr{C}\left(\mathbf{V M}_{1}\right) \subseteq \mathscr{C}(\mathbf{W})$ and hence we can write (41) as

$$
\mathscr{C}\left(\mathbf{V} \mathbf{M}_{1}\right) \subseteq \mathscr{C}\left(\dot{\mathbf{M}}_{1} \mathbf{X}_{2}\right)^{\perp} \cap \mathscr{C}(\mathbf{W}) .
$$

In view of part (e) of Lemma 1.2 we have the following:

$$
\mathscr{C}\left(\mathbf{X}_{1}: \mathbf{V M}\right)=\mathscr{C}\left(\dot{\mathbf{M}}_{1} \mathbf{X}_{2}: \mathbf{Q} \mathbf{W}\right)^{\perp}=\mathscr{C}\left(\dot{\mathbf{M}}_{1} \mathbf{X}_{2}\right)^{\perp} \cap \mathscr{C}(\mathbf{W}) .
$$

Combining (42) and (43) yields

$$
\mathscr{C}\left(\mathbf{V M}_{1}\right) \subseteq \mathscr{C}\left(\mathbf{X}_{1}: \mathbf{V M}\right),
$$

which is obviously equivalent to $\mathscr{C}\left(\mathbf{V M}_{1}\right)=\mathscr{C}(\mathbf{V M})$.

From Theorems 4.1 and 4.2 we get the following result.

Corollary 4.1. The following statements are equivalent:

(a) $\left\{\operatorname{BLUP}\left(\mathbf{M}_{1} \mathbf{X}_{2} \mathbf{u} \mid \mathscr{M}\right)\right\}=\left\{\operatorname{BLUE}\left(\mathbf{M}_{1} \mathbf{X}_{2} \boldsymbol{\beta}_{2} \mid \mathscr{F}\right)\right\}$,

(b) $\mathscr{C}\left(\mathbf{X}_{2}\right) \subseteq \mathscr{C}\left(\mathbf{X}_{1}: \mathbf{\Sigma} \mathbf{M}_{1} \mathbf{Q}_{\mathbf{M}_{1} \mathbf{V}}\right)$ and $\mathscr{C}\left(\mathbf{V M}_{1}\right)=\mathscr{C}(\mathbf{V M})$. 


\section{One further equality between BLUE and BLUP}

In this section we consider

$\operatorname{BLUE}\left(\mathbf{X}_{1} \boldsymbol{\beta}_{1}+\mathbf{X}_{2} \boldsymbol{\beta}_{2} \mid \mathscr{F}\right)$ versus $\operatorname{BLUP}\left(\mathbf{X}_{1} \boldsymbol{\beta}_{1}+\mathbf{X}_{2} \mathbf{u} \mid \mathscr{M}\right)$.

Theorem 5.1. An arbitrary BLUP for $\boldsymbol{\eta}=\mathbf{X}_{1} \boldsymbol{\beta}_{1}+\mathbf{X}_{2} \mathbf{u}$ under $\mathscr{M}$ provides also the BLUE for $\boldsymbol{\mu}=\mathbf{X}_{1} \boldsymbol{\beta}_{1}+\mathbf{X}_{2} \boldsymbol{\beta}_{2}$ under the fixed model $\mathscr{F}$, i.e.,

$\left\{\operatorname{BLUP}\left(\mathbf{X}_{1} \boldsymbol{\beta}_{1}+\mathbf{X}_{2} \mathbf{u} \mid \mathscr{M}\right)\right\} \subseteq\left\{\operatorname{BLUE}\left(\mathbf{X}_{1} \boldsymbol{\beta}_{1}+\mathbf{X}_{2} \boldsymbol{\beta}_{2} \mid \mathscr{F}\right)\right\}$,

i.e., $\left\{\mathbf{P}_{\boldsymbol{\eta} \mid \mathscr{M}}\right\} \subseteq\left\{\mathbf{P}_{\boldsymbol{\mu} \mid \mathscr{F}}\right\}$, if and only if

$$
\left\{\operatorname{BLUP}\left(\mathbf{M}_{1} \mathbf{X}_{2} \mathbf{u} \mid \mathscr{M}\right)\right\} \subseteq\left\{\operatorname{BLUE}\left(\mathbf{M}_{1} \mathbf{X}_{2} \boldsymbol{\beta}_{2} \mid \mathscr{F}\right)\right\} .
$$

Proof. The general solution to

$$
\mathbf{T}\left(\mathbf{X}_{1}: \Sigma \mathbf{M}_{1}\right)=\left(\mathbf{X}_{1}: \mathbf{X}_{2} \mathbf{D} \mathbf{X}_{2}^{\prime} \mathbf{M}_{1}\right)
$$

can be expressed as

$$
\begin{aligned}
\mathbf{T}_{0} & =\mathbf{X}_{1}\left(\mathbf{X}_{1}^{\prime} \mathbf{W}_{m}^{-} \mathbf{X}_{1}\right)^{-} \mathbf{X}_{1}^{\prime} \mathbf{W}_{m}^{+}+\mathbf{X}_{2} \mathbf{D} \mathbf{X}_{2}^{\prime} \mathbf{M}_{1}\left(\mathbf{M}_{1} \boldsymbol{\Sigma} \mathbf{M}_{1}\right)^{+} \mathbf{M}_{1}+\mathbf{E} \mathbf{Q}_{\mathbf{W}_{m}} \\
& =\mathbf{G}_{\boldsymbol{\mu}_{1} \mid \mathscr{M}}+\mathbf{G}_{\mathbf{X}_{2} \mathbf{u} \mid \mathscr{M}}+\mathbf{E} \mathbf{Q}_{\mathbf{W}_{m}},
\end{aligned}
$$

where $\mathbf{E}$ is free to vary and $\mathbf{W}_{m}=\boldsymbol{\Sigma}+\mathbf{X}_{1} \mathbf{X}_{1}^{\prime}$. Suppose that $\mathbf{T}_{0}$ provides also the BLUE for $\boldsymbol{\mu}=\mathbf{X} \boldsymbol{\beta}$ under the fixed model $\mathscr{F}$. Then $\mathbf{T}_{0}$ has to satisfy, for every $\mathbf{E}$, the fundamental BLUE equation

$$
\mathbf{T}_{0}\left(\mathbf{X}_{1}: \mathbf{X}_{2}: \mathbf{V M}\right)=\left(\mathbf{X}_{1}: \mathbf{X}_{2}: \mathbf{0}\right) \text {. }
$$

It is obvious that the $\mathbf{X}_{1}$-part of (46) holds. Moreover, we must have

$$
\left(\mathbf{G}_{\boldsymbol{\mu}_{1} \mid \mathscr{M}}+\mathbf{G}_{\mathbf{X}_{2} \mathbf{u} \mid \mathscr{M}}+\mathbf{E Q}_{\mathbf{W}_{m}}\right)\left(\mathbf{X}_{2}: \mathbf{V M}\right)=\left(\mathbf{X}_{2}: \mathbf{0}\right) \text { for all } \mathbf{E},
$$

from which it follows that $\mathscr{C}\left(\mathbf{X}_{2}\right) \subseteq \mathscr{C}\left(\mathbf{W}_{m}\right)$ and that for some $\mathbf{A}$ and $\mathbf{B}$,

$$
\mathbf{X}_{2}=\mathbf{X}_{1} \mathbf{A}+\boldsymbol{\Sigma} \mathbf{M}_{1} \mathbf{B}
$$

We further must have

$$
\left(\mathbf{G}_{\boldsymbol{\mu}_{1} \mid \mathscr{M}}+\mathbf{G}_{\mathbf{X}_{2} \mathbf{u} \mid \mathscr{M}}\right)\left(\mathbf{X}_{2}: \mathbf{V M}\right)=\left(\mathbf{X}_{2}: \mathbf{0}\right) .
$$

It is straightforward to show that $\left(\mathbf{G}_{\boldsymbol{\mu}_{1} \mid \mathscr{M}}+\mathbf{G}_{\mathbf{X}_{2} \mathbf{u} \mid \mathscr{M}}\right) \mathbf{V M}=\mathbf{0}$, so that we are left with condition

$$
\begin{aligned}
\left(\mathbf{G}_{\boldsymbol{\mu}_{1} \mid \mathscr{M}}+\mathbf{G}_{\mathbf{X}_{2} \mathbf{u} \mid \mathscr{M}}\right) \mathbf{X}_{2}= & \mathbf{X}_{1}\left(\mathbf{X}_{1}^{\prime} \mathbf{W}_{m}^{-} \mathbf{X}_{1}\right)^{-} \mathbf{X}_{1}^{\prime} \mathbf{W}_{m}^{+} \mathbf{X}_{2} \\
& +\mathbf{X}_{2} \mathbf{D} \mathbf{X}_{2}^{\prime} \mathbf{M}_{1}\left(\mathbf{M}_{1} \boldsymbol{\Sigma} \mathbf{M}_{1}\right)^{-} \mathbf{M}_{1} \mathbf{X}_{2}=\mathbf{X}_{2} .
\end{aligned}
$$

Substituting $\mathbf{X}_{2}=\mathbf{X}_{1} \mathbf{A}+\boldsymbol{\Sigma} \mathbf{M}_{1} \mathbf{B}=\mathbf{X}_{1} \mathbf{A}+\mathbf{W}_{m} \mathbf{M}_{1} \mathbf{B}$ into 48 gives

$$
\mathbf{X}_{1} \mathbf{A}+\mathbf{X}_{2} \mathbf{D} \mathbf{X}_{2}^{\prime} \mathbf{M}_{1} \mathbf{B}=\mathbf{X}_{1} \mathbf{A}+\mathbf{\Sigma} \mathbf{M}_{1} \mathbf{B}
$$

so that we have $\mathbf{X}_{2} \mathbf{D} \mathbf{X}_{2}^{\prime} \mathbf{M}_{1} \mathbf{B}=\mathbf{\Sigma} \mathbf{M}_{1} \mathbf{B}$, i.e., $\mathbf{V M}_{1} \mathbf{B}=\mathbf{0}$ and thereby

$$
\mathscr{C}(\mathbf{B}) \subseteq \mathscr{C}\left(\mathbf{M}_{1} \mathbf{V}\right)^{\perp}
$$

Combining (47) and 49 gives $\mathscr{C}\left(\mathbf{X}_{2}\right) \subseteq \mathscr{C}\left(\mathbf{X}_{1}: \mathbf{\Sigma} \mathbf{M}_{1} \mathbf{Q}_{\mathbf{M}_{1} \mathbf{V}}\right)$, and thus by Theorem 4.1 the proof is completed. 
Consider now the reverse inclusion of (44).

Theorem 5.2. An arbitrary BLUE for $\boldsymbol{\mu}=\mathbf{X}_{1} \boldsymbol{\beta}_{1}+\mathbf{X}_{2} \boldsymbol{\beta}_{2}$ under $\mathscr{F}$ provides also the BLUP for $\boldsymbol{\eta}=\mathbf{X}_{1} \boldsymbol{\beta}_{1}+\mathbf{X}_{2} \mathbf{u}$ under the mixed model $\mathscr{M}$, i.e.,

$$
\left\{\operatorname{BLUE}\left(\mathbf{X}_{1} \boldsymbol{\beta}_{1}+\mathbf{X}_{2} \boldsymbol{\beta}_{2} \mid \mathscr{F}\right)\right\} \subseteq\left\{\operatorname{BLUP}\left(\mathbf{X}_{1} \boldsymbol{\beta}_{1}+\mathbf{X}_{2} \mathbf{u} \mid \mathscr{M}\right)\right\},
$$

i.e., $\left\{\mathbf{P}_{\boldsymbol{\mu} \mid \mathscr{F}}\right\} \subseteq\left\{\mathbf{P}_{\boldsymbol{\eta} \mid \mathscr{M}}\right\}$, if and only if

$$
\left\{\operatorname{BLUE}\left(\mathbf{M}_{1} \mathbf{X}_{2} \boldsymbol{\beta}_{2} \mid \mathscr{F}\right)\right\} \subseteq\left\{\operatorname{BLUP}\left(\mathbf{M}_{1} \mathbf{X}_{2} \mathbf{u} \mid \mathscr{M}\right)\right\} .
$$

Proof. Take an arbitrary member in the class $\left\{\mathbf{P}_{\boldsymbol{\mu} \mid \mathscr{F}}\right\}$,

$$
\mathbf{G}_{0}=\mathbf{G}+\mathbf{E} \mathbf{Q}_{\mathbf{W}}=\mathbf{X}\left(\mathbf{X}^{\prime} \mathbf{W}^{-} \mathbf{X}\right)^{-} \mathbf{X}^{\prime} \mathbf{W}^{+}+\mathbf{E} \mathbf{Q}_{\mathbf{W}},
$$

where $\mathbf{E}$ is free to vary and $\mathscr{C}(\mathbf{W})=\mathscr{C}\left(\mathbf{X}_{1}: \mathbf{X}_{2}: \mathbf{V}\right)$. Then $\mathbf{G}_{0}$ provides the BLUP for $\boldsymbol{\eta}=\mathbf{X}_{1} \boldsymbol{\beta}_{1}+\mathbf{X}_{2} \mathbf{u}$ under the mixed model $\mathscr{M}$ if and only if

$$
\left(\mathbf{G}+\mathbf{E} \mathbf{Q}_{\mathbf{W}}\right)\left(\mathbf{X}_{1}: \mathbf{\Sigma} \mathbf{M}_{1}\right)=\left(\mathbf{X}_{1}: \mathbf{X}_{2} \mathbf{D} \mathbf{X}_{2}^{\prime} \mathbf{M}_{1}\right) .
$$

The $\mathbf{X}_{1}$-part in 50$)$ is clear. The $\mathbf{\Sigma}_{1}$-part gives

$$
\mathbf{G} \boldsymbol{\Sigma} \mathbf{M}_{1}=\mathbf{X}\left(\mathbf{X}^{\prime} \mathbf{W}^{-} \mathbf{X}\right)^{-} \mathbf{X}^{\prime} \mathbf{W}^{+} \boldsymbol{\Sigma} \mathbf{M}_{1}=\mathbf{X}_{2} \mathbf{D} \mathbf{X}_{2}^{\prime} \mathbf{M}_{1} .
$$

Premultiplying (51) by $\mathbf{X}^{\prime} \mathbf{W}^{+}$gives an equivalent form

$$
\mathbf{X}^{\prime} \mathbf{W}^{+} \boldsymbol{\Sigma} \mathbf{M}_{1}=\mathbf{X}^{\prime} \mathbf{W}^{+} \mathbf{X}_{2} \mathbf{D} \mathbf{X}_{2}^{\prime} \mathbf{M}_{1} \text {. }
$$

Substituting $\mathbf{X}_{2} \mathbf{D} \mathbf{X}_{2}^{\prime}=\boldsymbol{\Sigma}-\mathbf{V}$ into 52 leads to

$$
\mathbf{X}^{\prime} \mathbf{W}^{+} \boldsymbol{\Sigma} \mathbf{M}_{1}=\mathbf{X}^{\prime} \mathbf{W}^{+}(\boldsymbol{\Sigma}-\mathbf{V}) \mathbf{M}_{1},
$$

i.e., $\mathbf{X}^{\prime} \mathbf{W}^{+} \mathbf{V M}_{1}=\mathbf{0}$, i.e.,

$$
\mathscr{C}\left(\mathbf{V M}_{1}\right) \subseteq \mathscr{C}\left(\mathbf{W}^{+} \mathbf{X}\right)^{\perp} .
$$

Now by part (d) of Lemma 1.2 we know that

$$
\mathscr{C}\left(\mathbf{W}^{+} \mathbf{X}\right)^{\perp}=\mathscr{C}\left(\mathbf{W} \mathbf{M}: \mathbf{Q}_{\mathbf{W}}\right)=\mathscr{C}\left(\mathbf{V M}: \mathbf{Q}_{\mathbf{W}}\right),
$$

and hence 53 becomes

$$
\mathscr{C}\left(\mathbf{V M}_{1}\right) \subseteq \mathscr{C}\left(\mathbf{V M}: \mathbf{Q}_{\mathbf{W}}\right)
$$

Premultiplying (54) by $\mathbf{P}_{\mathbf{W}}$ we obtain $\mathscr{C}\left(\mathbf{V M}_{1}\right) \subseteq \mathscr{C}(\mathbf{V M})$, so that we must have $\mathscr{C}\left(\mathbf{V M}_{1}\right)=\mathscr{C}(\mathbf{V M})$, and thus by Theorem 4.2 the proof is completed.

Combining the theorems of Sections 4 and 5 we get the following interesting result.

Corollary 5.1. The following statements are equivalent:

(a) $\left\{\operatorname{BLUP}\left(\mathbf{X}_{1} \boldsymbol{\beta}_{1}+\mathbf{X}_{2} \mathbf{u} \mid \mathscr{M}\right)\right\}=\left\{\operatorname{BLUE}\left(\mathbf{X}_{1} \boldsymbol{\beta}_{1}+\mathbf{X}_{2} \boldsymbol{\beta}_{2} \mid \mathscr{F}\right)\right\}$,

(b) $\left\{\operatorname{BLUP}\left(\mathbf{M}_{1} \mathbf{X}_{2} \mathbf{u} \mid \mathscr{M}\right)\right\}=\left\{\operatorname{BLUE}\left(\mathbf{M}_{1} \mathbf{X}_{2} \boldsymbol{\beta}_{2} \mid \mathscr{F}\right)\right\}$,

(c) $\mathscr{C}\left(\mathbf{X}_{2}\right) \subseteq \mathscr{C}\left(\mathbf{X}_{1}: \mathbf{\Sigma} \mathbf{M}_{1} \mathbf{Q}_{\mathbf{M}_{1} \mathbf{V}}\right)$ and $\mathscr{C}\left(\mathbf{V M}_{1}\right)=\mathscr{C}(\mathbf{V M})$. 


\section{Equality of the covariance matrices}

In this section we assume that $\boldsymbol{\mu}_{1}=\mathbf{X}_{1} \boldsymbol{\beta}_{1}$ is estimable under $\mathscr{F}$ and we consider the equality of the covariance matrices of the BLUEs of $\boldsymbol{\mu}_{1}$ under $\mathscr{F}$ and under $\mathscr{M}$, i.e., we are comparing $\operatorname{cov}\left(\mathbf{G}_{\boldsymbol{\mu}_{1} \mid \mathscr{M}} \mathbf{\mathbf { y }} \mid \mathscr{M}\right)$ and $\operatorname{cov}\left(\mathbf{G}_{\boldsymbol{\mu}_{1} \mid \mathscr{F}} \mathbf{y} \mid \mathscr{F}\right)$, where

$$
\begin{aligned}
\mathbf{G}_{\boldsymbol{\mu}_{1} \mid \mathscr{F}} & =\mathbf{X}_{1}\left(\mathbf{X}_{1}^{\prime} \dot{\mathbf{M}}_{2} \mathbf{X}_{1}\right)^{-} \mathbf{X}_{1}^{\prime} \dot{\mathbf{M}}_{2} \in\left\{\mathbf{P}_{\boldsymbol{\mu}_{1} \mid \mathscr{F}}\right\}, \\
\mathbf{G}_{\boldsymbol{\mu}_{1} \mid \mathscr{M}} & =\mathbf{X}_{1}\left(\mathbf{X}_{1}^{\prime} \mathbf{W}_{m}^{+} \mathbf{X}_{1}\right)^{-} \mathbf{X}_{1}^{\prime} \mathbf{W}_{m}^{+} \in\left\{\mathbf{P}_{\boldsymbol{\mu}_{1} \mid \mathscr{M}}\right\} .
\end{aligned}
$$

It is noteworthy that the covariance matrices of the BLUEs are unique even though the representations of the BLUEs may not be unique.

It can be shown, see, e.g., [13, that

$$
\begin{aligned}
\operatorname{cov}\left(\mathbf{G}_{\boldsymbol{\mu}_{1} \mid \mathscr{M}} \mathbf{y} \mid \mathscr{M}\right) & =\mathbf{G}_{\boldsymbol{\mu}_{1} \mid \mathscr{M}} \boldsymbol{\Sigma}_{\mathbf{G}_{\boldsymbol{\mu}_{1} \mid \mathscr{M}}^{\prime}} \\
& =\mathbf{X}_{1}\left[\left(\mathbf{X}_{1}^{\prime} \mathbf{W}_{m}^{+} \mathbf{X}_{1}\right)^{+}-\mathbf{I}_{p_{1}}\right] \mathbf{X}_{1}^{\prime} \\
& =\mathbf{X}_{1}\left[\left(\mathbf{X}_{1}^{\prime} \mathbf{W}_{m}^{+1 / 2} \mathbf{W}_{m}^{+1 / 2} \mathbf{X}_{1}\right)^{+}-\mathbf{I}_{p_{1}}\right] \mathbf{X}_{1}^{\prime},
\end{aligned}
$$

where $\mathbf{W}_{m}^{+1 / 2}$ refers to the Moore-Penrose inverse of the nonnegative definite square root of $\mathbf{W}_{m}$, and

$$
\begin{aligned}
\operatorname{cov}\left(\mathbf{G}_{\left.\boldsymbol{\mu}_{1}|\mathscr{F}| \mathscr{F}\right)} \mathbf{y} \mathbf{G}_{\boldsymbol{\mu}_{1} \mid \mathscr{F} \mathbf{V} \mathbf{G}_{\boldsymbol{\mu}_{1} \mid \mathscr{F}}^{\prime}}\right. & =\mathbf{X}_{1}\left[\left(\mathbf{X}_{1}^{\prime} \dot{\mathbf{M}}_{2} \mathbf{X}_{1}\right)^{+}-\mathbf{I}_{p_{1}}\right] \mathbf{X}_{1}^{\prime} \\
& =\mathbf{X}_{1}\left\{\left[\mathbf{X}_{1}^{\prime} \mathbf{M}_{2}\left(\mathbf{M}_{2} \mathbf{W}_{m} \mathbf{M}_{2}\right)^{+} \mathbf{M}_{2} \mathbf{X}_{1}\right]^{+}-\mathbf{I}_{p_{1}}\right\} \mathbf{X}_{1}^{\prime} \\
& =\mathbf{X}_{1}\left[\left(\mathbf{X}_{1}^{\prime} \mathbf{W}_{m}^{+1 / 2} \mathbf{P}_{\mathbf{W}_{m}^{1 / 2} \mathbf{M}_{2}} \mathbf{W}_{m}^{+1 / 2} \mathbf{X}_{1}\right)^{+}-\mathbf{I}_{p_{1}}\right] \mathbf{X}_{1}^{\prime}
\end{aligned}
$$

The equality $\operatorname{cov}\left(\mathbf{G}_{\boldsymbol{\mu}_{1} \mid \mathscr{M}} \mathbf{y} \mid \mathscr{M}\right)=\operatorname{cov}\left(\mathbf{G}_{\boldsymbol{\mu}_{1} \mid \mathscr{F}} \mathbf{y} \mid \mathscr{F}\right)$ holds if and only if

$$
\begin{aligned}
\mathbf{X}_{1}\left(\mathbf{X}_{1}^{\prime} \mathbf{W}_{m}^{+1 / 2}\right. & \left.\mathbf{W}_{m}^{+1 / 2} \mathbf{X}_{1}\right)^{+} \mathbf{X}_{1}^{\prime} \\
& =\mathbf{X}_{1}\left(\mathbf{X}_{1}^{\prime} \mathbf{W}_{m}^{+1 / 2} \mathbf{P}_{\mathbf{W}_{m}^{1 / 2} \mathbf{M}_{2}} \mathbf{W}_{m}^{+1 / 2} \mathbf{X}_{1}\right)^{+} \mathbf{X}_{1}^{\prime} .
\end{aligned}
$$

Pre- and postmultiplying (55) by $\mathbf{X}_{1}^{+}$and $\left(\mathbf{X}_{1}^{\prime}\right)^{+}$, respectively, and using the fact that $\mathbf{P}_{\mathbf{X}_{1}^{\prime}}=\mathbf{X}_{1}^{+} \mathbf{X}_{1}$, gives an equivalent form to 55 :

i.e.,

$$
\left(\mathbf{X}_{1}^{\prime} \mathbf{W}_{m}^{+1 / 2} \mathbf{W}_{m}^{+1 / 2} \mathbf{X}_{1}\right)^{+}=\left(\mathbf{X}_{1}^{\prime} \mathbf{W}_{m}^{+1 / 2} \mathbf{P}_{\mathbf{W}_{m}^{1 / 2} \mathbf{M}_{2}} \mathbf{W}_{m}^{+1 / 2} \mathbf{X}_{1}\right)^{+},
$$

$$
\mathbf{X}_{1}^{\prime} \mathbf{W}_{m}^{+1 / 2} \mathbf{W}_{m}^{+1 / 2} \mathbf{X}_{1}=\mathbf{X}_{1}^{\prime} \mathbf{W}_{m}^{+1 / 2} \mathbf{P}_{\mathbf{W}_{m}^{1 / 2} \mathbf{M}_{2}} \mathbf{W}_{m}^{+1 / 2} \mathbf{X}_{1}
$$

Now we have the Löwner ordering

$$
\mathbf{X}_{1}^{\prime} \mathbf{W}_{m}^{+1 / 2}\left(\mathbf{I}_{n}-\mathbf{P}_{\mathbf{W}_{m}^{1 / 2} \mathbf{M}_{2}}\right) \mathbf{W}_{m}^{+1 / 2} \mathbf{X}_{1} \geq_{\mathbf{L}} \mathbf{0},
$$

where the equality holds if and only if

$$
\mathscr{C}\left(\mathbf{W}_{m}^{+1 / 2} \mathbf{X}_{1}\right) \subseteq \mathscr{C}\left(\mathbf{W}_{m}^{1 / 2} \mathbf{M}_{2}\right) .
$$


Premultiplying (56) by $\mathbf{W}_{m}^{1 / 2}$ gives an equivalent inclusion

$$
\mathscr{C}\left(\mathbf{X}_{1}\right) \subseteq \mathscr{C}\left(\mathbf{W}_{m} \mathbf{M}_{2}\right)=\mathscr{C}\left(\mathbf{W}_{1} \mathbf{M}_{2}\right) \text {, where } \mathbf{W}_{1}=\mathbf{X}_{1} \mathbf{X}_{1}^{\prime}+\mathbf{V} .
$$

As Isotalo et al. [11, p. 73] point out, the assumption $\mathscr{C}\left(\mathbf{W}_{m}\right)=\mathbb{R}^{n}$ implies that the BLUE of $\boldsymbol{\mu}_{1}$ has a unique representation under $\mathscr{F}$ and $\mathscr{M}$. Moreover, following their proof (assuming the estimability of $\boldsymbol{\mu}_{1}$ under $\mathscr{F}$ it can be shown that the presentations are equal if and only if (57) holds. Thus we can conclude the following result.

Theorem 6.1. The following statements are equivalent.

(a) $\operatorname{cov}\left(\mathbf{G}_{\boldsymbol{\mu}_{1} \mid \mathscr{M}} \mathbf{y} \mid \mathscr{M}\right)=\operatorname{cov}\left(\mathbf{G}_{\boldsymbol{\mu}_{1} \mid \mathscr{F}} \mathbf{y} \mid \mathscr{F}\right)$.

(b) $\mathscr{C}\left(\mathbf{X}_{1}\right) \subseteq \mathscr{C}\left(\mathbf{W}_{m} \mathbf{M}_{2}\right)$.

(c) If $\mathscr{C}\left(\mathbf{W}_{m}\right)=\mathbb{R}^{n}$, then the representations of the BLUEs of $\boldsymbol{\mu}_{1}$ under the models $\mathscr{F}$ and $\mathscr{M}$ are equal.

\section{Conclusions}

In this article we consider the partitioned fixed linear model $\mathscr{F}: \mathbf{y}=$ $\mathbf{X}_{1} \boldsymbol{\beta}_{1}+\mathbf{X}_{2} \boldsymbol{\beta}_{2}+\varepsilon$ and the corresponding mixed model $\mathscr{M}: \mathbf{y}=\mathbf{X}_{1} \boldsymbol{\beta}_{1}+$ $\mathbf{X}_{2} \mathbf{u}+\boldsymbol{\varepsilon}$, where $\boldsymbol{\varepsilon}$ is a random error vector and $\mathbf{u}$ is a random effect vector. Isotalo et al. [11] found conditions under which an arbitrary representation of the best linear unbiased estimator, BLUE, of $\boldsymbol{\theta}_{1}=\mathbf{M}_{2} \mathbf{X}_{1} \boldsymbol{\beta}_{1}$ in the fixed model $\mathscr{F}$ remains BLUE in the mixed model $\mathscr{M}$; here $\mathbf{M}_{2}$ refers to the orthogonal projector $\mathbf{I}_{n}-\mathbf{P}_{\mathbf{X}_{2}}$. The reason to concentrate on estimating $\boldsymbol{\theta}_{1}=\mathbf{M}_{2} \mathbf{X}_{1} \boldsymbol{\beta}_{1}$ is that this approach means that the properties obtained are valid for all parametric functions of the type $\mathbf{K}_{1} \boldsymbol{\beta}_{1}$ that are estimable under the partitioned model $\mathscr{F}$ (and thereby under $\mathscr{M}$ ). In this paper we extend the results concerning further equalities arising from the models $\mathscr{F}$ and $\mathscr{M}$.

The property that BLUE of $\boldsymbol{\theta}_{1}$ under $\mathscr{F}$ remains BLUE under $\mathscr{M}$ can be denoted shortly as

$$
\left\{\operatorname{BLUE}\left(\boldsymbol{\theta}_{1} \mid \mathscr{F}\right)\right\} \subseteq\left\{\operatorname{BLUE}\left(\boldsymbol{\theta}_{1} \mid \mathscr{M}\right)\right\},
$$

or, equivalently as $\left\{\mathbf{P}_{\boldsymbol{\theta}_{1} \mid \mathscr{F}}\right\} \subseteq\left\{\mathbf{P}_{\boldsymbol{\theta}_{1} \mid \mathscr{M}}\right\}$, where, in notation introduced in Section 1

$$
\begin{aligned}
& \mathbf{A} \in\left\{\mathbf{P}_{\boldsymbol{\theta}_{1} \mid \mathscr{F}}\right\} \Longleftrightarrow \mathbf{A}\left(\mathbf{X}_{1}: \mathbf{X}_{2}: \mathbf{V M}\right)=\left(\mathbf{M}_{2} \mathbf{X}_{1}: \mathbf{0}: \mathbf{0}\right), \\
& \mathbf{B} \in\left\{\mathbf{P}_{\boldsymbol{\theta}_{1} \mid \mathscr{M}}\right\} \Longleftrightarrow \mathbf{B}\left(\mathbf{X}_{1}: \mathbf{\Sigma} \mathbf{M}_{1}\right)=\left(\mathbf{M}_{2} \mathbf{X}_{1}: \mathbf{0}\right) .
\end{aligned}
$$

In this paper we generalize the results of [11] by considering the following relations:

$$
\begin{array}{rlll}
\operatorname{BLUE}\left(\mathbf{M}_{2} \mathbf{X}_{1} \boldsymbol{\beta}_{1} \mid \mathscr{F}\right) & \text { vs } & \operatorname{BLUP}\left(\mathbf{M}_{2} \mathbf{X}_{1} \boldsymbol{\beta}_{1}+\mathbf{X}_{2} \mathbf{u} \mid \mathscr{M}\right), \\
\operatorname{BLUE}\left(\mathbf{M}_{2} \mathbf{X}_{2} \boldsymbol{\beta}_{2} \mid \mathscr{F}\right) & \text { vs } & \operatorname{BLUP}\left(\mathbf{M}_{2} \mathbf{X}_{2} \mathbf{u} \mid \mathscr{M}\right), \\
\operatorname{BLUE}(\mathbf{X} \boldsymbol{\beta} \mid \mathscr{F}) & \text { vs } & \operatorname{BLUP}\left(\mathbf{X}_{1} \boldsymbol{\beta}_{1}+\mathbf{X}_{2} \mathbf{u} \mid \mathscr{M}\right) .
\end{array}
$$


As Kala et al. [14, Remark 2] point out, the notation of the type as in (58) is merely symbolic and it is not meant to refer to a set containing only one element which is a single fixed vector resulting from a transformation of an observed vector $\mathbf{y}$, or is a single random vector variable being a specific linear transformation of the random vector $\mathbf{y}$. We are, of course, actually interested in the matrices belonging to classes like $\left\{\mathbf{P}_{\boldsymbol{\theta}_{1} \mid \mathscr{F}}\right\}$ etc.

There are several related papers concerning the invariance of the BLUEs and/or BLUPs under two models. Mitra and Moore [18 gave an extensive study on the circumstances in which the BLUEs of estimable parametric functions of the fixed parameters in linear model $\left\{\mathbf{y}, \mathbf{X} \boldsymbol{\beta}, \mathbf{V}_{1}\right\}$ remain BLUEs under $\left\{\mathbf{y}, \mathbf{X} \boldsymbol{\beta}, \mathbf{V}_{2}\right\}$; models differing in covariance matrices. Corresponding considerations related to two mixed models have been made, e.g., by Haslett and Puntanen [5, 6]. In [7, they provide a review of conditions under which BLUEs/BLUPs in one linear mixed model are also BLUE/BLUPs in another. The article [8] explores interesting links between the mixed and fixed linear models. It appears that the concept of the linear model with new future observations is a powerful tool for these considerations. For further references we may mention [15], [22, [25], and [4].

We believe that our results, which are mainly linear-algebraic by nature, can provide some insight into the relations between the fixed and mixed model like $\mathscr{F}$ and $\mathscr{M}$. Some interesting related discussion appears, e.g., in [9, 10].

\section{Acknowledgements}

Many thanks go to an anonymous referee for constructive remarks.

\section{References}

[1] R. Christensen, Plane Answers to Complex Questions: the Theory of Linear Models, 4th Edition. Springer, New York, 2001.

[2] J. Groß and S. Puntanen, Estimation under a general partitioned linear model, Linear Algebra Appl. 321 (2000), 131-144.

[3] S. J. Haslett, J. Isotalo, Y. Liu, and S. Puntanen, Equalities between OLSE, BLUE and BLUP in the linear model, Stat. Pap. 55 (2014), 543-561.

[4] S. J. Haslett, X.-Q. Liu, A. Markiewicz, and S. Puntanen, Some properties of linear sufficiency and the BLUPs in the linear mixed model, Stat. Pap. 61 (2020), 385-401.

[5] S. J. Haslett and S. Puntanen, A note on the equality of the BLUP s for new observations under two linear models, Acta Comment. Univ. Tartu. Math. 14 (2010), 27-33.

[6] S. J. Haslett and S. Puntanen, On the equality of the BLUPs under two linear mixed models, Metrika 74 (2011), 381-395.

[7] S. J. Haslett and S. Puntanen, A review of conditions under which BLUEs and/or $\mathrm{BLUP} s$ in one linear mixed model are also BLUEs and/or BLUP $s$ in another, Calcutta Statist. Assoc. Bull. 65 (2013), 25-41.

[8] S. J. Haslett, S. Puntanen, and B. Arendacká, The link between the mixed and fixed linear models revisited, Stat. Pap. 56 (2015), 849-861. 
[9] F. K. C. Hui, S. Müller, and A. H. Welsh, Testing random effects in linear mixed models: another look at the F-test (with discussion), Aust. New Zealand J. Stat. 61 (2019), 61-84.

[10] F. K. C. Hui, S. Müller, and A. H. Welsh, Random effects misspecification can have severe consequences for random effects inference in linear mixed models, Int. Stat. Rev. 89 (2021), 186-206.

[11] J. Isotalo, M. Möls, and S. Puntanen, Invariance of the BLUE under the linear fixed and mixed effects models, Acta Comment. Univ. Tartu. Math. 10 (2006), 69-76.

[12] J. Isotalo and S. Puntanen, Linear prediction sufficiency for new observations in the general Gauss-Markov model, Commun. Stat. Theory Methods 35 (2006), 1011-1023.

[13] J. Isotalo, S. Puntanen, and G. P. H. Styan, The BLUE's covariance matrix revisited: a review, J. Stat. Plan. Inference 138 (2008), 2722-2737.

[14] R, Kala, S. Puntanen, and Y. Tian, Some notes on linear sufficiency, Stat. Pap. 58 (2017), 1-17.

[15] X. Liu and Q.-W. Wang, Equality of the BLUPs under the mixed linear model when random components and errors are correlated. J. Multivar. Anal. 116 (2013), 297-309.

[16] A. Markiewicz and S. Puntanen, Further properties of the linear sufficiency in the partitioned linear model, Matrices, Statistics and Big Data: Selected Contributions from IWMS 2016. S.E. Ahmed, F. Carvalho \& S. Puntanen, Eds. Springer, Cham, 2019, 1-22.

[17] G. Marsaglia and G. P. H. Styan, Equalities and inequalities for ranks of matrices, Linear Multilinear Algebra 2 (1974), 269-292.

[18] S. K. Mitra and B. J. Moore, Gauss-Markov estimation with an incorrect dispersion matrix, Sankhyā Ser. A 35 (1973), 139-152.

[19] S. Puntanen, G. P. H. Styan, and J. Isotalo, Matrix Tricks for Linear Statistical Models: Our Personal Top Twenty, Springer, Heidelberg, 2011.

[20] C. R. Rao, Representations of best linear estimators in the Gauss-Markoff model with a singular dispersion matrix, J. Multivariate Anal. 3 (1973), 276-292.

[21] C. R. Rao and S. K. Mitra, Generalized Inverse of Matrices and Its Applications. Wiley, New York, 1971.

[22] J.-Y. Rong and X.-Q. Liu, On misspecification of the dispersion matrix in mixed linear models, Stat. Pap. 51 (2010), 445-453.

[23] Y. Tian, A matrix handling of predictions of new observations under a general random-effects model, Electron. J. Linear Algebra 29 (2015), 30-45.

[24] Y. Tian, A new derivation of BLUPs under random-effects model, Metrika 78 (2015), 905-918.

[25] Q.-W. Wang and X. Liu, The equalities of BLUPs for linear combinations under two general linear mixed models. Commun. Stat. Theory Methods 42 (2013), 3528-3543.

School of Fundamental Sciences, Massey Univ., Palmerston North, New Zealand; Centre for Public Health Research, Massey Univ., Wellington, New Zealand; National Institute for Applied Statistics Research Australia, Univ. of Wollongong, Australia; Research School of Finance, Actuarial Studies and Statistics, The Australian National Univ., Canberra, Australia

E-mail address: s.j.haslett@massey.ac.nz

Faculty of Information Technology and Communication Sciences, FI-33014

TAMPERE UNIVERSITY, FinLAND

E-mail address: jarkko.isotalo@tuni.fi

E-mail address: simo.puntanen@tuni.fi 